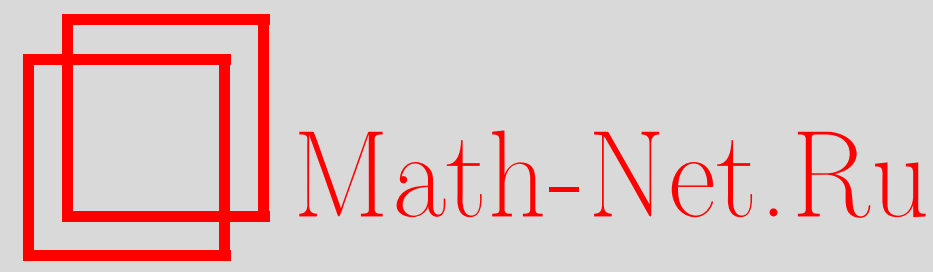

Е. М. Матвеев, Явная нижняя оценка однородной рациональной линейной формы от логарифмов алгебраических чисел, Изв. РАН. Сер. матем., 1998, том 62, выпуск 4, 81-136

DOI: https://doi.org/10.4213/im190

Использование Общероссийского математического портала Math-Net.Ru подразумевает, что вы прочитали и согласны с пользовательским соглашением

http://www. mathnet.ru/rus/agreement

Параметры загрузки:

IP : 54.197 .217 .227

26 апреля 2023 г., 13:07:47 
УДК 511

Е. М. Матвеев

\section{Явная нижняя оценка однородной рациональной линейной формы от логарифмов алгебраических чисел}

В работе изучается линейная форма вида $\Lambda=b_{1} \ln \alpha_{1}+\cdots+b_{n} \ln \alpha_{n}$ с целыми рациональными коэффициентами $b_{j}\left(b_{n} \neq 0, n \geqslant 2\right)$ и алгебраическими числами $\alpha_{j}$, удовлетворяющими условию так называемой сильной независимости. В стандартных обозначениях доказана явная оценка вида

$$
|\Lambda|>\exp \left(-C^{n} D^{n+2} \Omega \ln \left(C^{n} D^{n+2} \Omega^{\prime}\right) \ln (e B)\right)
$$

Новизна оценки состоит в отсутствии в ней множителя вида $n^{n}$. Библиографияя: 28 наименований.

\section{§1. Введение}

Пусть дано поле $\mathbb{K},[\mathbb{K}: \mathbb{Q}]=D_{\mathbb{K}}$, и числа $\beta_{0}, \ldots, \beta_{n} \in \mathbb{K}, \alpha_{1}, \ldots, \alpha_{n} \in$ $\mathbb{K}^{*}=\mathbb{K} \backslash\{0\}$. Теория линейных форм от логарифмов в общем случае имеет дело с выражениями вида

$$
\Lambda=\beta_{0}+\beta_{1} \ln \alpha_{1}+\cdots+\beta_{n} \ln \alpha_{n},
$$

а в так называемом однородном рациональном случае - с выражениями

$$
\Lambda=b_{1} \ln \alpha_{1}+\cdots+b_{n} \ln \alpha_{n},
$$

где $b_{j}=\beta_{j} \in \mathbb{Z}$.

Мы будем всегда предполагать, что $\Lambda \neq 0, \beta_{n} \neq 0$. В общем случае мы имеем $n \geqslant 1$, а в однородном рациональном случае $n \geqslant 2$.

В течение последнего времени эта тема подвергалась тщательному исследованию как в виду ее многочисленных приложений, так и вследствие плодотворных идей, появившихся в этой теории и связанных в значительной мере с именами А. О. Гельфонда, создавшего основу метода, и А. Бейкера, предложившего решающее усиление. Краткую историю вопроса до 1976 г. можно найти в работе [1], где также были представлены явные оценки:

$$
|\Lambda|>\exp \left(-C_{1} \Omega\left(\ln \Omega^{\prime}\right) \ln (B \Omega)\right)
$$

Работа частично поддержана индивидуальным грантом фонда Сороса.

(C) E. M. Матвеeb 1998 
в общем случае и

$$
|\Lambda|>\exp \left(-C_{1} \Omega\left(\ln \Omega^{\prime}\right) \ln B\right)
$$

в однородном рациональном случае. Здесь использованы обозначения

$$
\begin{aligned}
A_{j} & \geqslant \max \left\{4, \ln H\left(\alpha_{j}\right)\right\}, \quad 1 \leqslant j \leqslant n, \\
\Omega & =A_{1} \ldots A_{n}, \quad \Omega^{\prime}=A_{1} \ldots A_{n-1}, \quad A_{1} \leqslant \cdots \leqslant A_{n}, \\
B & \geqslant \max \left\{4, H\left(\beta_{1}\right), \ldots, H\left(\beta_{n}\right)\right\}, \quad C_{1}=(16 n D)^{200 n}, \quad D=D_{\mathbb{K}},
\end{aligned}
$$

а $H(\alpha)$ обозначает обычную высоту алгебраического числа $\alpha$, т.е. максимум модулей коэффициентов его минимального многочлена над $\mathbb{Z}$. Бейкер также предполагал, что взяты главные значения логарифмов.

ЗАмечАнИЕ. Другие авторы понимают под числами $A_{j}$ экспоненты наших $A_{j}$ и вынуждены писать $\ln A_{j}$, где мы пишем $A_{j}$.

Было замечено, что в случае так называемой сильной независимости $\alpha_{1}, \ldots, \alpha_{n}$ может быть получена значительно лучшая константа $C_{1}$, а общий случай может быть сведен к нему с некоторой потерей точности. Явная оценка линейной формы (1.2) при условии сильной независимости, лучшая, чем у Бейкера, была получена в [2]. Эта оценка также была в терминах обычных высот.

В данной работе мы будем понимать под условием сильной независимости равенство

$$
\left[\mathbb{K}\left(\sqrt{\alpha_{1}}, \ldots, \sqrt{\alpha_{n}}\right): \mathbb{K}\right]=2^{n}
$$

Иногда берутся другие показатели корня. В первых работах это было обусловлено техникой доказательства. Позднее было замечено, что достаточно квадратного корня, при этом получаются лучшие оценки. Другие значения могут быть полезны в $p$-адическом случае. Предположение (1.5) позволяет применять теорию Куммеpa, оно упрошает финальное рассуждение в доказательстве и улучшает зависимость оценки от $n$. Поэтому мы также будем называть (1.5) условием Куммера (или, более корректно, что числа $\alpha_{1}, \ldots, \alpha_{n}$ удовлетворяют условию Куммера относительно поля $\mathbb{K})$. Впервые оно было введено Бейкером и Старком в [3].

В 1980 г. появилась работа [4], где константа $C_{1}$ из (1.3) стала иметь вид $C_{1}=D^{n+2} C_{2}(n)$. Константа $C_{2}$ в общем случае равна $n^{2 n} 2^{8 n+51}$, а при условии (1.5) $C_{2}=n^{n+4} 2^{9 n+26}$. (Также появилась некоторая зависимость от $n, D$ под знаком логарифма в $\ln \Omega^{\prime}$.)

С тех пор зависимость от $D$ не менялась. Отметим, что такого рода зависимость при $n=2$ была установлена еше Гельфондом в 1952 г. (см. [5, п. 3.4, теорема 3]). Даже странно, что в обшем случае это было сделано столь поздно. Одно из объяснений того, что Вальдшмит сумел получить такую зависимость от $D$, а также 
улучшить остальные константы, заключается в том, что он работал не с логарифмами обычных высот, а с логарифмическими высотами (определение см. в $\S 3$ ), и в его обозначениях было

$$
\begin{gathered}
A_{j} \geqslant \max \left\{h\left(\alpha_{j}\right), \frac{\left|\ln \alpha_{j}\right|}{D}, \frac{1}{D}\right\}, \quad 1 \leqslant j \leqslant n ; \\
\ln B \geqslant \max \left\{h\left(\beta_{0}\right), \ldots, h\left(\beta_{n}\right)\right\} .
\end{gathered}
$$

Стоит отметить, что оценка Вальдшмита зависит также еще от одного параметра, который может быть выражен с помошью величины

$$
E=\max \left\{\frac{\left|\ln \alpha_{j}\right|}{D A_{j}}: 1 \leqslant j \leqslant n\right\} .
$$

Чем меньше $E$, тем лучше будет оценка для $|\Lambda|$. Зависимость от этого параметра в методе Гельфонда-Бейкера была впервые изучена Т. Шореем в [6]. Отметим, правда, что очень малое $E$ встречается редко, и если кто-либо захочет получить примеры с хорошей оценкой для $|\Lambda|$ в силу малости $E$, то будет проще использовать технику, основанную на свойствах так называемых $G$-функций, дающую здесь лучшие оценки, чем метод Гельфонда-Бейкера (см. [7] для рациональных $\alpha_{j}$ и $[8]$ в общем случае). Конечно, в вычислительных приложениях линейных форм от логарифмов следует учитывать любое усиление. Большинство поздних оценок также учитывают этот параметр. В данной работе мы тоже вводим параметр такого типа, и наша зависимость будет лучше предшествуюших.

Оценка Вальдшмита относилась только к формам (1.1), и если кто-либо хотел оценивать формы (1.2), то он вынужден был рассматривать их как частный случай (1.1), хотя ясно, что оценка для форм (1.2) могла бы быть лучше. В теоретических и практических приложениях однородный рациональный случай более важен, чем общий (см., например, [9], [10]). У Вальдшмита константа $C_{2}$ была сравнительно большой, но уже такой величины, что стали возможны некоторые компютерные вычисления с использованием оценок линейных форм от логарифмов. Так возникла проблема вывода новой явной оценки $|\Lambda|$ для однородного рационального случая.

В 1983 г. автор анонсировал в [11] оценку для случая сильной независимости, имевшую вид

$$
\begin{gathered}
|\Lambda|>\exp \left(-C_{2} D^{n+2} \Omega \ln \left(e^{5} D^{2} A_{n-1}\right) \ln \left(e^{2} B\right)\right), \\
C_{2}=e^{8} n^{n+4.5}\left(2 e^{3}\right)^{n} .
\end{gathered}
$$

Если $\mathbb{K}$ комплексное, то $D$ может быть заменено на $D / 2$. Константа $C_{2}$ лучше, чем у Вальдшмита, но при этом в (1.7) также улучшена зависимость от чисел $b_{j}$, поскольку в (1.7) параметр $B$ равен

$$
B=\max \left\{\frac{\left|b_{j}\right| A_{j}}{A_{n}}: 1 \leqslant j \leqslant n\right\},
$$


в то время как обычное определение $B$ из (1.6) дает

$$
B=\max \left\{\left|b_{j}\right|: 1 \leqslant j \leqslant n\right\} .
$$

K сожалению, автор не опубликовал доказательства (1.7). Это в основном было вызвано попытками доказать подобньй результат без предположения (1.5), но успеха в этом направлении добиться не удалось. Когда удаляется условие (1.5), то константа $C_{2}$ сильно увеличивается, появляется новый множитель $n^{n}$, а $B$ вида (1.8) нужно заменить на $B$ вида (1.9). В то же время отметим, что при получении оценок типа (1.7) трудно сосчитать константу $C_{2}$, тогда как учет комплексности $\mathbb{K}$ и замена зависимости от $B$ вида (1.9) на $B$ вида (1.8) делается путем небольшого изменения обозначений в стандартном доказательстве. В данной работе зависимость от $B$ будет даже немного точнее, чем (1.8). Это также будет получено путем небольшого изменения обозначений.

В приложениях оценок линейных форм от логарифмов выяснилось, что в наиболее практически важных случаях условие (1.5) выполнено. Стало настоятельно необходимо иметь хорошие оценки в однородном рациональном случае даже при ограничении (1.5). Дважды различные авторы (см. [12]) в 1987 г. и (см. [13]) в 1990 г. возвращались к этой проблеме. Используя [4] как образец с изменением некоторых обозначений и параметров, они получали оценки с константами, как у Вальдшмита, и с зависимостью от $B$ типа (1.8), так что их константа $C_{2}$ была хуже, чем в (1.7).

Из других улучшений оценок линейных форм от логарифмов следует выделить независимые результаты Вюстхольца [14] и Филиппона с Вальдшмитом [15], представленных на конференции в Дархэме в 1986 г., в которых из оценок был удален множитель $\ln \Omega^{\prime}$. Этот успех стал возможен благодаря новым оценкам кратностей нулей для алгебраических групп, полученным методами алгебраической геометрии. Однако технические трудности в этих доказательствах были столь велики, что до сих пор еше нет явных оценок, где бы при этом не ухудшалась бы зависимость от $n$ или $B$. Так, в [12] и в [13] множитель $\ln \Omega^{\prime}$ был оставлен, хотя эти работы и были после Дархэма.

Очень точная оценка для $n=2$ была получена в [16]. Хотя зависимость от $B$ была вида $\ln ^{2} B$, но константа $C_{2}$ была весьма малой, так что в приложениях, где все параметры невелики, эта оценка лучше других. Доказательство было основано на методе Шнайдера, а не Бейкера. Эта оценка неоднократно усиливалась.

В недавней работе Вальдшмита [17], содержашей новую явную оценку формы (1.2), множитель $\ln \Omega^{\prime}$ тоже удален. Отличительной особенностью этого результата является отсутствие условия (1.5) и зависимость от $B$ типа (1.9). Ценой этого улучшения явилось появление в оценке множителя $n^{3 n}$. Доказательство основано на модифицированном методе Шнайдера. 
Другая недавняя явная оценка $|\Lambda|$ в однородном рациональном случае принадлежит Бейкеру и Вюстхольцу [18]. Авторам удалось совместить новые оценки кратности нулей с теорией Куммера и получить оценку $|\Lambda|$ без множителя $\ln \Omega^{\prime}$ и ухудшения зависимости от $n$. Их константа $C_{1}$ имеет вид

$$
C_{1}=18(n+1) ! n^{n+1}(32 D)^{n+2} \ln (2 n D) .
$$

Отметим, что их оценка относится к случаю без условия (1.5). Хотя авторы и свели его к случаю сильной независимости, но соответствующая ему явная оценка не была приведена. Ясно, что переход к обшему случаю дает лишний множитель $2^{1-n} n$ ! и ухудшает зависимости от $B$. В [18] зависимость от $B$ имеет вид (1.9). Отметим, что в приложениях крайне важно иметь зависимость (1.8), а не (1.9). Так, в [19] авторы предпочли использовать работу [17], а не [18], хотя зависимость от $n$ там значительно хуже.

В данной работе мы представляем новую явную оценку линейных форм от логарифмов в однородном рациональном случае с условием Куммера. Эта оценка сильнее анонсированной в [11]. Главное усиление состоит в устранении множителя $n^{n}$ из $C_{2}$. Отметим, что в практических приложениях этот множитель наиболее неприятен (см., например, [9], [10]). Как уже отмечалось, такой же множитель появляется при устранении условия (1.5). Теперь он становится доминируюшим, поэтому мы не доказываем оценку для общего случая. Для его полного устранения нужны новые идеи.

Еще одно усиление лежит в зависимости от $\left|\ln \alpha_{j}\right|$, которая будет значительно слабее, чем в (1.6). Мы также убираем нижнюю границу $1 / D$ для $A_{j}$ путем включения более слабых условий.

Наша оценка слабее вышеприведенных в том плане, что она содержит множитель типа $\ln \Omega^{\prime}$. Он не является неустранимым. Автор полагает, что он может быть удален тем же способом, что и в [18], но не знает, насколько ухудшится при этом зависимость от $n$ и $B$.

\section{§2. Формулировки результатов}

На протяжении всей статьи мы сохраняем обозначения: $\mathbb{K}$ - алгебраическое поле степени $D_{\mathbb{K}}$ над $\mathbb{Q}$, вложенное в $\mathbb{C}$. Если $\mathbb{K} \subseteq \mathbb{R}$, то положим $\varkappa=1$, иначе $\varkappa=2, D=D_{\mathbb{K}} / \varkappa$. Даны числа $\alpha_{1}, \ldots, \alpha_{n} \in \mathbb{K}^{*}, n \geqslant 2$, удовлетворяющие условию Куммера и имеюшие абсолютные логарифмические высоты $h\left(\alpha_{j}\right)$, $1 \leqslant j \leqslant n, \ln \alpha_{1}, \ldots, \ln \alpha_{n}-$ произвольные данные значения логарифмов. Положим

$$
\rho=\operatorname{rank}_{\mathbb{R}}\left\{\ln \alpha_{1}, \ldots, \ln \alpha_{n}\right\} .
$$

Рассматривается линейная форма

$$
\Lambda=b_{1} \ln \alpha_{1}+\cdots+b_{n} \ln \alpha_{n}
$$


c $b_{1}, \ldots, b_{n} \in \mathbb{Z}, b_{n} \neq 0$.

Будем использовать положительные числа $A_{1}, \ldots, A_{n}$ (напомним, что они соответствуют $\left(\ln A_{j}\right)$ в работах других авторов). Положим

$$
\begin{aligned}
\vartheta & \geqslant \frac{1}{n}\left(\frac{h\left(\alpha_{1}\right)}{A_{1}}+\cdots+\frac{h\left(\alpha_{n}\right)}{A_{n}}\right), \\
E & \geqslant \frac{1}{n D} \max \left\{\left| \pm \frac{\ln \alpha_{1}}{A_{1}} \pm \cdots \pm \frac{\ln \alpha_{n}}{A_{n}}\right|\right\}, \\
E_{1} & =\frac{1}{(n-1) D \vartheta}\left(\frac{1}{A_{1}}+\cdots+\frac{1}{A_{n}}\right) .
\end{aligned}
$$

Зададим параметры $C_{1}, C_{2}, C_{3}, C_{3}^{*}$ условиями

$$
\begin{gathered}
C_{3}^{*} \exp \left(C_{3}^{*}\right) \frac{E e}{2 \vartheta} \geqslant e^{n / \rho}, \quad C_{3}=\max \left\{C_{3}^{*}, \frac{n}{\rho}\right\} . \\
C_{1}=\left(1+\frac{e^{-2 n}}{148}\right)(n \ln 2+2) \frac{\left(1+\frac{1}{n}\right)}{C_{3}} \\
C_{2}=4(n+1)\left(6+\frac{5}{n \ln 2+2}\right) \frac{e^{2 n}}{n^{1 / 2} C_{3}} .
\end{gathered}
$$

Положим для краткости

$$
\Omega=A_{1} \ldots A_{n}, \quad \omega=\Omega\left(\frac{C_{1} D \vartheta}{e}\right)^{n}\left(C_{3} \exp \left(C_{3}\right) \frac{E e}{2 \vartheta}\right)^{\rho}
$$

Пусть $C_{0}$ удовлетворяет условию

$$
C_{0} \geqslant \max \left\{2 C_{3}, \ln \left(C_{2} D \max \left\{\frac{C_{0} \omega}{C_{1} A_{n} \vartheta D}, C_{0}, \frac{2 C_{3} E_{1}}{C_{1}}\right\}\right)\right\} .
$$

Положим также

$$
\begin{aligned}
B_{0} & =\sum_{j=1}^{n-1} \frac{\left|b_{n}\right|+\left|b_{j}\right|}{\operatorname{HОД~}\left(b_{j}, b_{n}\right) 2 D \omega C_{0} C_{2}}, \\
B_{1} & =\sum_{j=1}^{n-1} \frac{1}{\operatorname{HОД}\left(b_{j}, b_{n}\right) 2 C_{1} n D \vartheta}\left(\frac{\left|b_{n}\right|}{A_{j}}+\frac{\left|b_{j}\right|}{A_{n}}\right), \\
B_{2} & =\sum_{j=1}^{n-1} \frac{\left|\ln \alpha_{j}\right|\left(\left|b_{n}\right|+\left|b_{j}\right|\right)}{\left|b_{n}\right| 2 D \omega C_{0} C_{2}} \\
B_{3} & =\sum_{j=1}^{n-1} \frac{\left|\ln \alpha_{j}\right|}{2 C_{1}\left|b_{n}\right| D \vartheta n}\left(\frac{\left|b_{n}\right|}{A_{j}}+\frac{\left|b_{j}\right|}{A_{n}}\right)
\end{aligned}
$$


и пусть

$$
W_{0} \geqslant \max \left\{2 C_{3}, \ln \left(e\left(1+B_{0}+B_{1}+B_{2}+B_{3}\right)\right)\right\}
$$

TEOPEMA 2.1. Если

$$
\begin{gathered}
\frac{D \omega \min \left\{C_{0}, W_{0}\right\}}{2 C_{3}} \geqslant 1, \\
\frac{\omega}{C_{1} \vartheta A_{j}} \frac{\min \left\{C_{0}, W_{0}\right\}}{2 C_{3}} \geqslant 1, \quad 1 \leqslant j \leqslant n, \\
3\left(C_{1} D \vartheta\right)^{n-1} \frac{D \Omega}{A_{j}}\left(C_{3} \exp \left(C_{3}\right) \frac{E e}{2 \vartheta}\right)^{\rho-1} \frac{C_{0}}{C_{3}} \geqslant 1, \quad 1 \leqslant j \leqslant n,
\end{gathered}
$$

mo

$$
\ln |\Lambda|>-91 \cdot 2^{n} C_{2} C_{0} D^{2} W_{0} \omega
$$

Следующая теорема упрощает утверждение теоремы 2.1. Она позволяет проводить сравнение с предыдушими результатами и применима в случаях, не требуюших особо точных оценок.

TEOPEMA 2.2. Пусть $\vartheta=1, E=1, C_{3}=n / \rho$,

$$
\begin{gathered}
A_{j}=\max \left\{h\left(\alpha_{j}\right), \frac{\left|\ln \alpha_{j}\right|}{D}, \frac{1}{D C_{1}}\right\}, \quad 1 \leqslant j \leqslant n, \\
B=\max \left\{\frac{\left|b_{j}\right| A_{j}}{A_{n}}: 1 \leqslant j \leqslant n\right\}, \\
C_{0}^{\prime}=\ln \left(\frac{C_{2} D \omega}{C_{1} A_{n}}\right) .
\end{gathered}
$$

Тогда

$$
\ln |\Lambda|>-112 \cdot 2^{n} C_{2} C_{0}^{\prime} D^{2} \omega \ln (2 e B)
$$

Отметим, что множитель, зависящий от $n$, равен здесь

$$
112 \cdot 2^{n} C_{2} \vartheta^{n} C_{1}^{n} e^{-n}\left(C_{3} \exp \left(C_{3}\right) \frac{E e}{2 \vartheta}\right)^{\rho}<2^{15}\left(\frac{n e}{2 \rho}\right)^{\rho} \frac{\rho\left(2 e^{2} \rho \ln 2\right)^{n}}{n^{1 / 2}}
$$

Теорема 2.3. Пусть $\mathbb{K}=\mathbb{Q}, A_{j} \geqslant h\left(\alpha_{j}\right), \quad 1 \leqslant j \leqslant n, \quad E=1, C_{3}=n$, $C_{0}, W_{0}, B, C_{0}^{\prime}$ удовлетворяют $(2.6),(2.8),(2.14),(2.15)$. Тогда:

(i) выполняются неравенства (2.12), (2.16) с $\vartheta=1$;

(ii) если $\alpha_{j} \in \mathbb{Z}, \alpha_{j}>0, A_{j}=\ln \alpha_{j}, \quad 1 \leqslant j \leqslant n$, то выполняются неравенства (2.12), (2.16) с $\vartheta=\frac{1}{2}\left(1-1 / n e^{n+1}\right)$;

(iii) для указанного $\vartheta$ в (2.6) выполняется $D \omega /\left(C_{1} A_{n} \vartheta\right)>E_{1} / C_{1}$. 
ЗАмечаниЕ 2.1. Случай $\varkappa=2, \rho=1$ не редок, что видно из следующего примера: для алгебраического $0<\beta<1$ выполняется

$$
\left|b_{1} \pi+b_{2} \arcsin \beta\right|=\left|b_{1} \ln \alpha_{1}+b_{2} \ln \alpha_{2}\right|,
$$

где $\alpha_{1}=-1$ и $\alpha_{2}$ удовлетворяет уравнению $\alpha_{2}^{2}-2 i \beta \alpha_{2}-1=0$.

ЗАмЕчАниЕ 2.2. Случай $\varkappa=1, \rho=2$ можно исключить, так как если $\mathbb{K} \subseteq \mathbb{R}$, то для $\alpha \in \mathbb{K}$ также будет $|\alpha|= \pm \alpha \in \mathbb{K}$, и мы имеем

$$
\left|b_{1} \ln \alpha_{1}+\cdots+b_{n} \ln \alpha_{n}\right| \geqslant\left|b_{1} \ln \right| \alpha_{1}\left|+\cdots+b_{n} \ln \right| \alpha_{n}||
$$

так что можно брать только положительные $\alpha_{j}$ и главные значения логарифма.

ЗАмЕчАНИЕ 2.3. Зависимость от $\varkappa$ была указана в [11], зависимость от $\rho$ вводится впервые; если $\varkappa=2, \rho=1$, то оценка будет лучше.

ЗАмечАниЕ 2.4 . Зависимость $(2.7)$ от $b_{j}$ новая, поскольку учитывает возможное наличие нетривиального НОД $\left(b_{j}, b_{n}\right)$. Она может быть полезна, например, при изучении уравнения Каталана, где появляется форма вида

$$
\pm p \ln p \pm q \ln q+p q \ln \left(\frac{u}{v}\right) .
$$

ЗАмЕчАниЕ 2.5. Нижние границы в условиях (2.9)-(2.11), равно как и нижние гранищы для $C_{0} / C_{3}, W_{0} / C_{3}$, являются техническими. Если мы заменим их на другие значения, то это может ухудшить оценки $|\Lambda|$, но не настолько, как если бы мы просто увеличили соответствующие параметры, чтобы удовлетворять исходным условиям. Соответствуюшие изменения легко могут быть сделаны в $\S 11,12$. Эти условия выбраны, потому что случай $\mathbb{K}=\mathbb{Q}$ им удовлетворяет.

ЗАмечание 2.6. Легко видеть, что условия (2.1)-(2.11) однородны по $A_{1}, \ldots, A_{n}$, т.е. если мы умножим все $A_{j}$ на некоторое $\lambda>0$ и разделим $\vartheta, E$ на $\lambda$, то все эти условия останутся верными. Варьируя $A_{1}, \ldots, A_{n}$, можно добиться некоторой оптимизации, которая, грубо говоря, означает, что при фиксированном $\vartheta$ и равенстве в (2.1) следует минимизировать $E$ из (2.2). Но на самом деле можно получить дальнейшие усиления, если принять во внимание соображения, изложенные в $\S 5$.

ЗАмЕчАниЕ 2.7. Случай (ii) теоремы 2.3 усиливает общий случай на множитель порядка $2^{n}$. Здесь $\vartheta$ может не удовлетворять условию (2.1).

ЗАмЕчАниЕ 2.8. Случай (ii) теоремы 2.3 допускает дальнейшие усиления, которые могут быть сделаны, если мы оценим объем тела $\mathscr{W}$ более точно (см. $\S 5$ ). Аналогично, параметр $\vartheta$ из теоремы 2.1 может быть определен более точно, чем в (2.1). Соответствуюшее выражение для $\vartheta$ можно найти в $\S 5$. Здесь мы использовали его простейшую оценку, чтобы не утяжелять формулировку теоремы. 


\section{§3. Обозначения и соответствующие леммы}

Мы имеем $D_{\mathbb{K}}$ вложений $\mathbb{K}$ в $\mathbb{C}$, соответственно имеется $D_{\mathbb{K}}$ архимедовских нормирований поля $\mathbb{K}$ :

$$
|\alpha|_{\sigma}=\left|\alpha^{(\sigma)}\right|, \quad 1 \leqslant \sigma \leqslant D_{\mathbb{K}}
$$

Для комплексно-сопряженных вложений соответствуюшие нормирования равны, но для удобства будем брать их оба. Будем отождествлять $\mathbb{K}$ с его вложением для $\sigma=1$. Пронумеруем простые идеалы поля $\mathbb{K}$ индексами $\sigma>D_{\mathbb{K}}$ и определим неархимедовские нормирования $\mathbb{K}$ по формуле

$$
|\alpha|_{\sigma}=(\operatorname{Norm} \mathfrak{p})^{-k}, \quad k=\operatorname{ord}_{\mathfrak{p}}(\alpha), \quad \mathfrak{p}=\mathfrak{p}_{\sigma}, \quad \sigma>D_{\mathbb{K}}, \quad \alpha \in \mathbb{K}^{*}
$$

Для заданных подобным образом нормирований выполняется формула произведения

$$
\prod_{\sigma=1}^{\infty}|\alpha|_{\sigma}=1, \quad \alpha \in \mathbb{K}^{*}
$$

Отметим также, что $|\alpha|_{\sigma} \neq 1$ только для конечного количества $\sigma$.

Если для некоторого ненулевого целого $m$ выполняется $\gamma^{m} \in \mathbb{K}$, то мы можем определить $|\gamma|_{\sigma}, \sigma \geqslant 1$, и $\ln \gamma^{(\sigma)}, 1 \leqslant \sigma \leqslant D$, не расширяя основного поля $\mathbb{K}$, по формулам

$$
|\gamma|_{\sigma}=\left|\gamma^{m}\right|_{\sigma}^{1 / m}, \quad \ln \gamma^{(\sigma)}=m^{-1} \ln \left(\gamma^{m}\right)^{(\sigma)}
$$

Для таких $\gamma$ также выполняется формула произведения.

Обычно $\sigma \mid \infty$ обозначает, что $\sigma$ архимедовское, а $\sigma \nmid \infty$ обозначает, что $\sigma$ неархимедовское, в последнем случае $\sigma \mid p$ обозначает, что идеал $\mathfrak{p}_{\sigma}$ делит идеал $(p)$. Нумерация нормирований удобна, когда не важна их специфика, например при суммировании по всем $\sigma$. Полагая, что $\mathbb{K}_{\sigma}-$ метрическое замыкание $\mathbb{K}$ в нормировании $\sigma$, введем обозначения:

$$
\varkappa_{\sigma}=\left[\mathbb{K}_{\sigma}: \mathbb{Q}_{\sigma}\right], \quad \sigma \geqslant 1, \quad \varkappa=\varkappa_{1},
$$

так что $\varkappa_{\sigma}=1$ для действительных и $\varkappa_{\sigma}=2$ для комплексных $\sigma$.

Локальные и абсолютная логарифмические высоты набора чисел $\mathscr{B}=$ $\left\{\beta_{j} \in \mathbb{K}: j \in \mathscr{J}\right\}$ определяются следуюшим образом:

$$
\begin{gathered}
h_{\sigma}(\mathscr{B})=\ln \max \left\{\left|\beta_{j}\right|_{\sigma}: j \in \mathscr{J}\right\}, \quad \sigma \geqslant 1, \\
h(\mathscr{B})=\frac{1}{D_{\mathbb{K}}} \sum_{\sigma=1}^{\infty} h_{\sigma}(\mathscr{B})
\end{gathered}
$$


(полагаем, что $\ln 0=-\infty)$. Абсолютную логарифмическую высоту числа $\alpha \in \mathbb{K}$ зададим следующим образом:

$$
h(\alpha)=h(\{1, \alpha\})=\frac{1}{D_{\mathbb{K}}} \sum_{\sigma=1}^{\infty} \max \left\{0, \ln |\alpha|_{\sigma}\right\}=\left.\frac{1}{2 D_{\mathbb{K}}} \sum_{\sigma=1}^{\infty}|\ln | \alpha\right|_{\sigma} \mid .
$$

Абсолютная логарифмическая высота числа $\alpha$ не зависит от поля, его содержащего. Отметим, что $h(\alpha)=0$ только для корней из 1 . Для остальных $\alpha \neq 0$ мы имеем $h(\alpha) \geqslant C(\mathbb{K})>0$. Для фиксированного поля $\mathbb{K}$ эта константа может быть явно найдена, но в общем случае вопрос еше не решен. В [20] дана сводка результатов по этой теме.

Мы будем использовать векторы с различными способами нумерации координат. Для некоторых из них зафиксируем обозначения:

1) $\bar{u}=\left(u_{1}, \ldots, u_{n}\right)-$ с помошью $\left(^{-}\right)$,

2) $\tilde{u}=\left(u_{1}, \ldots, u_{n-1}\right)-$ с помошью $\left({ }^{\sim}\right)$,

3) $\bar{u}^{\prime}=\left(u_{0}, \ldots, u_{n}\right)-$ с помощью $\left(^{-\prime}\right)$,

4) $\tilde{u}^{\prime}=\left(u_{0}, \ldots, u_{n-1}\right)-$ с помощью $\left(^{\sim \prime}\right)$.

Другие типы векторов мы будем обозначать через $\bar{u}^{\prime \prime}$, попутно объясняя порядок нумерации их координат, например:

5) $\bar{u}^{\prime \prime}=\left(u_{1}, \ldots, u_{J}\right)-$ с помошью $\left({ }^{-\prime \prime}\right)$.

Координаты векторов всегда будут обозначаться той же буквой, что и сам вектop.

Для действительных векторов $\bar{u}, \bar{v}$ мы будем понимать под $\bar{u} \geqslant \bar{v}$ (соответственно $\bar{u}>\bar{v}$ ) соответствующее неравенство для каждой координаты. Введем также обозначение

$$
|\bar{u}|=\left|u_{1}\right|+\cdots+\left|u_{n}\right|
$$

Если у нас есть скалярная функция $f(x) \neq|x|$ одной скалярной переменной (она может зависеть от других числовых аргументов), то мы определим вектор $f(\bar{u})$ для вектора $\bar{u}$ следуюшим образом:

$$
f(\bar{u})=\left(f\left(u_{1}\right), \ldots, f\left(u_{n}\right)\right) .
$$

Для векторов $\bar{u}, \bar{v}$ зададим скалярное произведение формулой

$$
\bar{u} \cdot \bar{v}=u_{1} v_{1}+\cdots+u_{n} v_{n}
$$

а для скалярной функции $f(x, y) \neq x y$ и векторов $\bar{u}, \bar{v}$ положим

$$
f(\bar{u}, \bar{v})=\prod_{j=1}^{n} f\left(u_{j}, v_{j}\right)
$$


например, нашу линейную форму (1.2) мы можем записать в виде

$$
\Lambda=\bar{b} \cdot \ln \bar{\alpha}
$$

а $\bar{\alpha}$ означает

$$
\bar{\alpha}^{\bar{l}}=\prod_{j=1}^{n} \alpha_{j}^{l_{j}} .
$$

Будем здесь говорить, что $\bar{l}-$ мульти-показатель $\bar{\alpha}$.

Эти обозначения сохраняются для всех типов векторов с учетом того, что диапазон суммирования соответствует типу участвующих векторов (для двух векторов он должен быть один и тот же).

Следующие обозначения относятся только к векторам первого типа, при этом получаются векторы второго типа. Положим

$$
\tilde{\chi}(\bar{u}, \bar{b})=\tilde{\chi}(\bar{u})=\left(\chi_{1}, \ldots, \chi_{n-1}\right), \quad \tilde{\nu}(\bar{u}, \bar{b})=\tilde{\nu}(\bar{u})=\left(\nu_{1}, \ldots, \nu_{n-1}\right)
$$

где координаты определены следуюшим образом:

$$
\begin{array}{cc}
\chi_{j}=u_{j} b_{n}-u_{n} b_{j}, & 1 \leqslant j \leqslant n-1, \\
\nu_{j}=\frac{u_{j} b_{n}-u_{n} b_{j}}{\operatorname{HОД}\left(b_{j}, b_{n}\right)}, & 1 \leqslant j \leqslant n-1 .
\end{array}
$$

Мы можем опускать аргумент $\bar{b}$, предполагая, что $\bar{b}$ взят из линейной формы (1.2) и фиксирован раз и навсегда. Отметим также, что $\tilde{\chi}$ определен для любого типа $\bar{b}$, но $\tilde{\nu}$ - только для целых $\bar{b}$. Смысл введения $\tilde{\nu}$ состоит в том, что все $b_{j}$ могут быть взаимно просты в совокупности, но не попарно.

Отметим очевидное, но полезное свойство векторов $\tilde{\chi}, \tilde{\nu}$ :

$$
\tilde{\chi}(\bar{u}+x \bar{b})=\tilde{\chi}(\bar{u}), \quad \tilde{\nu}(\bar{u}+x \bar{b})=\tilde{\nu}(\bar{u})
$$

Зарезервируем символы $j, k, l, m, s, t, \lambda, \tau, M$ за целозначными переменными. Кроме того, $j, m, s, t, \tau, M$ будут неотрицательны. В дальнейшем мы часто будем опускать подобные примечания. Множество натуральных (целых положительных) чисел будем обозначать через $\mathbb{N}$, а множество неотрицательных целых чисел - через $\mathbb{N}_{0}$.

Укажем одно равенство, которое можно получить, раскрьвая скобки в выражении $|\bar{a}|^{M}$ по биномиальной формуле:

$$
\frac{|\bar{a}|^{M}}{M !}=\sum_{|\bar{m}|=M} \frac{\bar{a}^{\bar{m}}}{\bar{m} !^{\overline{1}}},
$$


предполагая $\bar{a} \geqslant \overline{0}$ и $\overline{1}=(1, \ldots, 1)$. Если в сумме справа мы возьмем только одно слагаемое, то получим неравенство

$$
\frac{|\bar{a}|^{|\bar{m}|}}{|\bar{m}| !} \geqslant \frac{\bar{a}^{\bar{m}}}{\bar{m} ! \overline{1}}
$$

Следующее тривиальное утверждение сформулируем в виде леммы.

ЛЕмма 3.1. Пусть $f_{j}(z, m)$ и $g_{j}(z, m)$ - некоторые функиии, $1 \leqslant j \leqslant n-1$, $m=0,1, \ldots$, удовлетворяющие соотношениям

$$
f_{j}\left(z_{j}, m_{j}\right)=\sum_{\tau_{j}=0}^{m_{j}} c_{j}\left(m_{j}, \tau_{j}\right) g_{j}\left(z_{j}, \tau_{j}\right), \quad m_{j} \geqslant 0, \quad 1 \leqslant j \leqslant n-1,
$$

с некоторыми $c_{j}\left(m_{j}, \tau_{j}\right)$. Тогда функиии

$$
F(\tilde{z}, \widetilde{m})=\prod_{j=1}^{n-1} f_{j}\left(z_{j}, m_{j}\right), \quad G(\tilde{z}, \widetilde{m})=\prod_{j=1}^{n-1} g_{j}\left(z_{j}, m_{j}\right)
$$

удовлетворяют соотношениям

$$
F(\tilde{z}, \tilde{m})=\sum_{\tilde{\tau} \leqslant \tilde{m}} C(\widetilde{m}, \tilde{\tau}) G(\tilde{z}, \tilde{\tau}), \quad G(\widetilde{m}, \tilde{\tau})=\prod_{j=1}^{n-1} c_{j}\left(m_{j}, \tau_{j}\right) .
$$

Теперь введем одно понятие, которое в некотором смысле обобщает понятие логарифмической высоты. Пусть даны вектор $\bar{\alpha} \in\left(\mathbb{K}^{*}\right)^{n}$ и множество $\mathscr{W} \subseteq \mathbb{R}^{n}$. Тогда положим

$$
\begin{aligned}
h_{\sigma}(\bar{\alpha}, \mathscr{W}) & =\sup \left\{\bar{w} \cdot \ln |\bar{\alpha}|_{\sigma}: \bar{w} \in \mathscr{W}\right\}, \quad \sigma \geqslant 1, \\
h(\bar{\alpha}, \mathscr{W}) & =\frac{1}{D_{\mathbb{K}}} \sum_{\sigma=1}^{\infty} h_{\sigma}(\bar{\alpha}, \mathscr{W}) .
\end{aligned}
$$

Если $\mathscr{W} \subseteq \mathbb{Z}^{n}$, то $h(\bar{\alpha}, \mathscr{W})=h\left(\left\{\bar{\alpha}^{\bar{w}}: \bar{w} \in \mathscr{W}\right\}\right)$, а если $\mathscr{W}$ - множество стандартных базисных векторов, то $h(\bar{\alpha}, \mathscr{W})=h(\{\bar{\alpha}\})$.

Лемма 3.2. (i) Eсли $\mathscr{V} \subseteq \mathscr{W}, \operatorname{mo} h(\bar{\alpha}, \mathscr{V}) \leqslant h(\bar{\alpha}, \mathscr{W})$.

(ii) $h(\bar{\alpha}, r \mathscr{W})=r h(\bar{\alpha}, \mathscr{W}), r>0$.

(iii) $h(\bar{\alpha}, \mathscr{V}+\mathscr{W})=h(\bar{\alpha}, \mathscr{V})+h(\bar{\alpha}, \mathscr{W})$.

(iv) $h(\bar{\alpha},\{\bar{v}\})=0, \quad \bar{v} \in \mathbb{R}^{n}$.

(v) $h(\bar{\alpha}, \mathscr{W}+\bar{v})=h(\bar{\alpha}, \mathscr{W}), \quad \bar{v} \in \mathbb{R}^{n}$.

(vi) $h(\bar{\alpha}, \mathscr{W})$ не зависит от выбора поля, содержащего числа $\alpha$.

ДокАЗАТЕльСтво. (i) очевидно; (ii), (iii) следуют из более аккуратных и также очевидных неравенств

$$
h_{\sigma}(\bar{\alpha}, r \mathscr{W})=r h_{\sigma}(\bar{\alpha}, \mathscr{W}), \quad h_{\sigma}(\bar{\alpha}, \mathscr{V}+\mathscr{W})=h_{\sigma}(\bar{\alpha}, \mathscr{V})+h_{\sigma}(\bar{\alpha}, \mathscr{W})
$$

для каждого $\sigma$; (iv) следует из формулы произведения; (v) следует из (iii) и (iv); (vi) аналогично свойству обычной логариффмической высоты и доказывается так же (см. [21, п. 3.1]). 


\section{§4. Общая схема доказательства}

Основная идея изучения линейных форм от логарифмов, восходящая к работам А. О. Гельфонда, заключается в одновременном рассмотрении двух вспомогательных функций. Одна из них должна обладать хорошими алгебраическими, а другая - хорошими аналитическими свойствами. В предположении о малости $|\Lambda|$ можно заменять одну функцию другой с небольшим поправочным членом для некоторого диапазона изменения переменных. Однако правильньй выбор вспомогательной функции является весьма трудной задачей. Здесь одна из основных идей в теории линейных форм от логарифмов принадлежит А. Бейкеру. Его вспомогательная функция имела вид

$$
G\left(\tilde{z}^{\prime}\right)=\sum^{(0)} P_{0}\left(z_{0}, \bar{l}\right) \prod_{j=1}^{n-1} \alpha_{j}^{\left(l_{j} b_{n}-l_{n} b_{j}\right) z_{j} / b_{n}}
$$

где $P_{0}\left(z_{0}, \bar{l}\right)$ - многочлены с неопределенными коэффициентами, а суммирование распространено на некоторое множество $\mathscr{L}_{0} \subseteq \mathbb{Z}^{n}$ индексов $\bar{l}$. (Точнее, полиномиальная часть функции была введена Н.И. Фельдманом в [22], а выбор $\mathscr{L}_{0}$ менялся неоднократно. Главное усиление данной работы также получено благодаря должному выбору множества $\mathscr{L}_{0}$.) После взятия частных производных, деления выражений на общий для всех слагаемых множитель и подстановки $z_{j}=z$ мы получаем набор функций

$$
G_{s}\left(z, \widetilde{m}^{\prime}\right)=\sum^{(s)} P_{s}^{\left(m_{0}\right)}(z, \bar{l}) \tilde{\chi}^{\tilde{m}}(\bar{l}) \tilde{\alpha}^{\tilde{\chi}(\bar{l}) z / b_{n}}
$$

Здесь $s=0$, в дальнейшем появятся аналогичные выражения и для других значений $s$. Мы будем использовать аналитические свойства этих функций. Одновременно мы будем рассматривать функции с хорошими алгебраическими свойствами, имеющие вид

$$
F_{s}\left(x, \widetilde{m}^{\prime}\right)=\sum^{(s)} P_{s}^{\left(m_{0}\right)}(x, \bar{l}) \tilde{\chi}^{\widetilde{m}}(\bar{l}) \bar{\alpha}^{\bar{l} x} .
$$

Доказательство теорем проходит индукцией по $s$. На нулевом шаге $s=0$ надо найти коэффициенты многочленов, не все равные нулю, чтобы выполнялись равенства

$$
F_{s}\left(x, \widetilde{m}^{\prime}\right)=0, \quad\left|\widetilde{m}^{\prime}\right| \leqslant M_{s}, \quad x \in \mathscr{X}_{s},
$$

для некоторых $M_{0}$ и $\mathscr{X}_{0}$,

$$
\mathscr{X}_{0}=\left\{0, \pm 1, \pm 2, \ldots, \pm X_{0}\right\}
$$

Это будет сделано в $\S 6$ с помощью аналога леммы Зигеля. 
Шаг индукции проводится следуюшим образом. Сначала мы получаем равенства

$$
F_{s}\left(\frac{x}{2}, \widetilde{m}^{\prime}\right)=0, \quad\left|\widetilde{m}^{\prime}\right| \leqslant M_{s+1}, \quad x \in \mathscr{X}_{s+1},
$$

для некоторых $M_{s+1} \leqslant M_{s}$ и $\mathscr{X}_{s+1}$, удовлетворяюших условиям

$$
\begin{gathered}
M_{s+1} \geqslant M_{s}+\left[-\frac{M_{0}}{2^{s+1}\left(1+\varepsilon_{0}\right)}\right]+1, \quad s \geqslant 0, \\
\mathscr{X}_{s}=\left\{ \pm 1, \pm 3, \ldots, \pm\left(2 X_{s}-1\right)\right\}, \quad s>0 .
\end{gathered}
$$

(Здесь и далее мы будем вводить малые параметры $\varepsilon$, значения которых будут определены позднее. Отметим, что $\mathscr{X}_{0}$ немного отличается от остальных $\mathscr{X}_{s}$.) Числа $X_{s}$ задаются формулой

$$
X_{s+1}=\left\{\begin{array}{ll}
2 X_{s}, & 0 \leqslant s \leqslant S_{1}, \\
X_{s}, & S_{1}<s,
\end{array} \quad S_{1}=\log _{2}\left(\frac{M_{0}}{2+2 \varepsilon_{0}}\right) .\right.
$$

Это будет сделано в $\S 10$ с помощью экстраполящионной техники применительно к функциям $G_{s}\left(z, \widetilde{m}^{\prime}\right)$ из (4.1).

Отметим, что при таком выборе $S_{1}$ при $s>S_{1}$ числа $X_{s}, M_{s}$ не меняются, что упрошает индукцию.

Теперь посмотрим, что следует из равенств (4.5). Положим

$$
x=2 y+1, \quad \bar{l}=2 \bar{\lambda}+\bar{\delta}, \quad \bar{\delta} \in\{0,1\}^{n}, \quad \bar{\lambda} \in \mathscr{L}_{s}(\bar{\delta}) .
$$

Теперь мы имеем

$$
0=F_{s}\left(\frac{x}{2}, \widetilde{m}^{\prime}\right)=\sum_{\bar{\delta}}\left(\sum_{\bar{\lambda}} P_{s}^{\left(m_{0}\right)}\left(\frac{x}{2}, \bar{l}\right) \tilde{\chi}^{\widetilde{m}}(\bar{l}) \bar{\alpha}^{\bar{\lambda} x+\bar{\delta} y}\right) \bar{\alpha}^{\bar{\delta} / 2}
$$

В этом месте доказательства мы используем условие Куммера. Оно означает, что числа $\bar{\alpha}^{\bar{\delta} / 2}, \bar{\delta} \in\{0,1\}^{n}$, линейно независимы над $\mathbb{K}$. Множители в $F_{s}\left(x / 2, \widetilde{m}^{\prime}\right)$, стоящие перед $\bar{\alpha} \bar{\delta} / 2,-$ из поля $\mathbb{K}$, значит, все они исчезают, и мы получаем из (4.5), что

$$
\begin{aligned}
& \sum_{\bar{\lambda}} P_{s}^{\left(m_{0}\right)}\left(\frac{x}{2}, 2 \bar{\lambda}+\bar{\delta}\right) \tilde{\chi}^{\widetilde{m}}(2 \bar{\lambda}+\bar{\delta}) \bar{\alpha}^{\bar{\lambda} x}=0, \\
& \left|\widetilde{m}^{\prime}\right| \leqslant M_{s+1}, \quad x \in \mathscr{X}_{s+1}, \quad \bar{\delta} \in\{0,1\}^{n} .
\end{aligned}
$$

Теперь возьмем $\bar{\delta}$, для которого не все многочлены обращаются в нуль, и положим

$$
\mathscr{L}_{s+1}=\mathscr{L}_{s}(\bar{\delta}), \quad P_{s+1}(z, \bar{\lambda})=P_{s}\left(\frac{z}{2}, 2 \bar{\lambda}+\bar{\delta}\right) .
$$


Поскольку мы имеем (4.8) для всех $\widetilde{m}^{\prime}$ с $\left|\widetilde{m}^{\prime}\right| \leqslant M_{s+1}$, то мы можем с помошью (4.8) и леммы 3.1 получить равенства

$$
\sum^{(s+1)} P_{s+1}^{\left(m_{0}\right)}(x, \bar{\lambda}) \tilde{\chi}^{\widetilde{m}}(\bar{\lambda}) \bar{\alpha}^{\bar{\lambda} x}=0 .
$$

Если мы обозначим левую часть через $F_{s+1}\left(x, \widetilde{m}^{\prime}\right)$, то получим (4.3) для $s+1$. Это доказьвает шаг индукции.

Обозначив выбранное на $s$-м шаге $\bar{\delta}$ через $\bar{\delta}_{s}$, можно для каждого $\bar{\lambda} \in \mathscr{L}_{s}$ восстановить исходное $\bar{l} \in \mathscr{L}_{0}$ по формуле

$$
\bar{l}=\bar{l}(\bar{\lambda})=2^{s} \bar{\lambda}+\bar{\lambda}_{s}, \quad \bar{\lambda}_{s}=\bar{\delta}_{0}+2 \bar{\delta}_{1}+\cdots+2^{s-1} \bar{\delta}_{s-1} .
$$

Мы использовали лемму 3.1 , чтобы свести выражения $\tilde{\chi}^{\widetilde{m}}(\bar{l}(\bar{\lambda}))$ к виду $\tilde{\chi}^{\widetilde{m}}(\bar{\lambda})$, но в дальнейшем будет удобнее не делать этого.

Теперь посмотрим, насколько далеко надо проводить индукцию.

Каждая координата векторов $\bar{l} \in \mathscr{L}_{s}$ меняется в диапазоне

$$
L_{s, j, 0} \leqslant l_{j} \leqslant L_{s, j, 1}, \quad L_{s, j} \geqslant L_{s, j, 1}-L_{s, j, 0}, \quad 1 \leqslant j \leqslant n,
$$

и из индуктивного шага легко видно, что

$$
L_{s+1, j} \leqslant \frac{L_{s, j}}{2}, \quad 1 \leqslant j \leqslant n, \quad 0 \leqslant s \leqslant S .
$$

Если взять $S>\log _{2}\left(L_{0, n}\right)$, то после $S$ шагов индукции диапазон для $l_{n}$ будет меньше 1 , т.е. суммирования по $l_{n}$ не будет. Идея избавиться от суммирования только по $l_{n}$ принадлежит А. ван дер Поортену [23].

Пусть теперь

$$
L_{0} \geqslant \max \left\{\operatorname{deg} P_{0}(z, \bar{l}): \bar{l} \in \mathscr{L}_{0}\right\}
$$

тогда мы можем выбрать некоторые $x_{0} \in \mathscr{X}_{S}$ и $m_{0} \leqslant L_{0} /\left|\mathscr{X}_{S}\right|$ так, что не все из чисел

$$
\eta(\bar{l})=P_{S}^{\left(m_{0}\right)}\left(x_{0}, \bar{l}\right), \quad \bar{l} \in \mathscr{L}_{S},
$$

обрашаются в нуль. Предположим, что вьполняется неравенство

$$
M_{S} \geqslant\left[L_{S, 1}\right]+\cdots+\left[L_{S, n-1}\right]+\frac{L_{0}}{\left|\mathscr{X}_{S}\right|} .
$$

Тогда из (4.3) для $s=S$ получаем уравнения

$$
\begin{gathered}
\sum_{l_{n-1}=L_{S, n-1,0}}^{L_{S, n-1,1}} \ldots \sum_{l_{1}=L_{S, 1,0}}^{L_{S, 1,1}} \prod_{j=1}^{n-1}\left(b_{n} l_{j}-b_{j} l_{n}\right)^{m_{j}} \eta(\bar{l})=0, \\
0 \leqslant m_{j} \leqslant\left[L_{S, j}\right], \quad 1 \leqslant j \leqslant n-1 .
\end{gathered}
$$

Одной из новых черт нашего доказательства является то, что мы позволяем числам $L_{s, j, 0}$ и $L_{s, j, 1}$ быть зависимыми от значений $l_{j+1}, \ldots, l_{n}$, но числа $L_{s, j}$ будут от них независимы.

Лемма 4.1. Пусть $L_{S, j}$ имеют вид (4.10). Тогда система уравнений (4.12) не имеет ненулевого решения $\eta(\bar{l})$. 
ДоКАЗАТЕЛЬСТВО. Положим $\xi_{1}\left(l_{1}, \ldots, l_{n-1}\right)=\eta(\bar{l})$ и индуктивно определим числа

$$
\begin{aligned}
\xi_{j+1} & =\xi_{j+1}\left(m_{1}, \ldots, m_{j}, l_{j+1}, \ldots, l_{n-1}\right) \\
& =\sum_{l_{j}=L_{S, j, 0}}^{L_{S, j, 1}}\left(b_{n} l_{j}-b_{j} l_{n}\right)^{m_{j}} \xi_{j}, \quad 1 \leqslant j \leqslant n-1 .
\end{aligned}
$$

Видно, что набор чисел $\left\{\xi_{j+1}\right\}$ получается из набора $\left\{\xi_{j}\right\}$ с помощью матрищ Вандермонда, значит, если набор $\left\{\xi_{j}\right\}$ ненулевой, то таким же будет и набор $\left\{\xi_{j+1}\right\}$. Числа $\left\{\xi_{n}(\widetilde{m})\right\}$ являются левой частью (4.12), что доказывает лемму.

При выводе равенств (4.13) мы предполагали малость линейной формы, а лемма 4.1 дает противоречие с этим, что доказывает теорему об оценке линейной формы от логарифмов.

ЗАмЕчаниЕ 4.1. Мы выбрали наборы точек $\mathscr{X}_{s}$ из (4.4), (4.6), содержащие как положительные, так и отрицательные целые числа. Это позволяет существенно улучшить константы в оценках линейных форм. В работах [12], [13] этого не было сделано, именно поэтому оценки этих работ хуже, чем в [11].

\section{§5. Построение исходного набора мульти-показателей}

В этом и последующих параграфах мы будем вводить некоторые параметры, в терминах которых будут получены оценки линейных форм. В конце мы установим их соответствие с параметрами $\S 2$. Мы будем вводить параметры по мере необходимости, обосновывая такой выбор. Ввод некоторых из них превышает степень детализации теорем из $\S 2$ и может быть полезен для более аккуратных оценок, особенно для конкретных значений чисел $\alpha_{j}$.

Сначала мы опишем способ построения $\mathscr{L}_{0}$ - множества мульти-показателей в исходной вспомогательной функции $F_{0}\left(x, \widetilde{m}^{\prime}\right)$. Оно будет удовлетворять условию

$$
\mathscr{L}_{0} \subseteq\left(\mathscr{W}+\bar{w}_{0}\right) \cap \mathbb{Z}^{n}
$$

для некоторых $\mathscr{W} \subseteq \mathbb{R}^{n}$ и $\bar{w}_{0} \in \mathbb{R}^{n}$. Для этого мы возьмем некоторое $L>0$ и набор неотрицательных чисел $\left\{\vartheta_{0}, \vartheta_{1}, \ldots, \vartheta_{\sigma}, \ldots\right\}$, удовлетворяюших следуюшим условиям:

$1)$ если $\ln |\bar{\alpha}|_{\sigma}=\overline{0}$, то $\vartheta_{\sigma}=0$, другие $\vartheta_{\sigma}$ положительны $(\sigma \geqslant 1)$;

2) для комплексно сопряженных $\mathbb{K}^{(\sigma)}$ соответствующие $\vartheta_{\sigma}$ равны;

$3)$ если $\rho=1$, то $\vartheta_{1} \operatorname{Im}(\ln \bar{\alpha})=\vartheta_{0} \operatorname{Re}(\ln \bar{\alpha})\left(\right.$ в частности, если $\mathbb{K} \subseteq \mathbb{R}$, то $\left.\vartheta_{0}=0\right)$.

Отметим, что только конечное количество $\vartheta_{\sigma}$ отлично от нуля. 
Определим $\mathscr{W} \subseteq \mathbb{R}^{n}$ следуюшим образом: $\bar{w} \in \mathscr{W}$, если

$$
\begin{aligned}
& -\vartheta_{\sigma} L \leqslant \bar{w} \cdot \ln |\bar{\alpha}|_{\sigma} \leqslant \vartheta_{\sigma} L, \quad \sigma \geqslant 1, \\
& -\vartheta_{0} L \leqslant \bar{w} \cdot \operatorname{Im}(\ln \bar{\alpha}) \leqslant \vartheta_{0} L .
\end{aligned}
$$

Можно вводить и другие условия, играюшие роль в определении $\mathscr{W}$. Оно всегда будет ограниченным симметричным телом. Положим

$$
\begin{aligned}
L_{0, j, 0} & =L_{0, j, 0}\left(w_{j+1}, \ldots, w_{n}\right)=\inf \left\{w_{j}: \bar{w} \in \mathscr{W}\right\}, \\
L_{0, j, 1} & =L_{0, j, 1}\left(w_{j+1}, \ldots, w_{n}\right)=\sup \left\{w_{j}: \bar{w} \in \mathscr{W}\right\}, \\
L_{0, j} & =\sup \left\{L_{0, j, 1}-L_{0, j, 0}: w_{j+1}, \ldots, w_{n} \in \mathbb{R}\right\}, \quad 1 \leqslant j \leqslant n .
\end{aligned}
$$

В настоящее время обычный подход заключается в выборе некоторых положительных чисел $A_{1}, \ldots, A_{n}$ таких, чтобы далее положить

$$
L_{0, j, 0}=-\frac{L}{2 A_{j}}, \quad L_{0, j, 1}=\frac{L}{2 A_{j}}, \quad L_{0, j}=\frac{L}{A_{j}}, \quad 1 \leqslant j \leqslant n .
$$

Тело $\mathscr{W}$ определяется условиями: если $\bar{w} \in \mathscr{W}$, то

$$
L_{0, j, 0} \leqslant w_{j} \leqslant L_{0, j, 1}, \quad 1 \leqslant j \leqslant n .
$$

Тогда равенства (5.2), (5.3) можно рассматривать в качестве определений для чисел $\vartheta_{\sigma}$. Так мы будем поступать, если нет дополнительной информации о числах $\alpha$. В частных случаях для описания множества $\mathscr{W}$ можно использовать все условия (5.2)-(5.6). Таким способом мы можем улучшить зависимость оценки $|\Lambda|$ от чисел $\alpha_{j}$.

Главная идея, позволяюшая убрать множитель $n^{n}$ из оценки $|\Lambda|$, состоит в рассмотрении неравенства

$$
|\bar{w} \cdot \ln \bar{\alpha}| \leqslant \vartheta_{0}^{*} L, \quad \bar{w} \in \mathscr{W},
$$

не как определяюшего число $\vartheta_{0}^{*}$, а как принимаюшего участие в определении множества $\mathscr{W}$ при соответствуюшем выборе $\vartheta_{0}^{*}$ (в действительном случае $\vartheta_{1}=\vartheta_{0}^{*}$ ).

Возьмем $\vartheta$, удовлетворяющее условию

$$
\vartheta \geqslant \frac{1}{L n} h(\bar{\alpha}, \mathscr{W})=\frac{1}{n D_{\mathbb{K}}} \sum_{\sigma=1}^{\infty} \vartheta_{\sigma} .
$$

Следует стремиться найти для данных $\vartheta, \vartheta_{0}^{*}$ тело $\mathscr{W}$ максимального объема, удовлетворяюшее (5.7), (5.8). Это довольно трудная задача. Для наших целей мы используем следующую лемму.

ЛЕмма 5.1. Пусть даны симметричное выпуклое тело $\mathscr{W}_{0} \subseteq \mathbb{R}^{n}$, вектор $\bar{v} \in \mathbb{C}^{n}(\bar{v} \neq \overline{0}) u \eta>0$. Положим $\rho=\operatorname{rank}_{\mathbb{R}}\{\operatorname{Re} \bar{v}, \operatorname{Im} \bar{v}\}$,

$$
\vartheta_{1}^{*}=\sup \left\{|\bar{w} \cdot \bar{v}|: \bar{w} \in \mathscr{W}_{0}\right\}, \quad \mathscr{E}=\left\{\bar{w} \in \mathbb{R}^{n}:|\bar{w} \cdot \bar{v}| \leqslant \vartheta_{1}^{*}\right\} .
$$

Тогда

$$
\operatorname{Vol}\left(\mathscr{W}_{0} \cap \eta \mathscr{E}\right) \geqslant \operatorname{Vol}\left(\mathscr{W}_{0}\right) \min \left\{1, \eta^{\rho}\right\}
$$


ДокАЗАТЕЛЬСТво. Если $\eta \geqslant 1$, то утверждение леммы очевидно. Пусть теперь $\eta<1$. Рассмотрим подпространства $\mathscr{V}=\mathbb{R} \operatorname{Re}(\bar{v})+\mathbb{R} \operatorname{Im}(\bar{v}), \quad \mathscr{V} \perp$ ортогонально к $\mathscr{V}$ в $\mathbb{R}^{n}$. Имеем $\operatorname{dim} \mathscr{V}=\rho, \operatorname{dim} \mathscr{V}^{\perp}=n-\rho$. Рассмотрим функцию

$$
f(\bar{w})=\operatorname{Vol}_{n-\rho}\left(\mathscr{W}_{0} \cap\left(\bar{w}+\mathscr{V}^{\perp}\right)\right), \quad \bar{w} \in \mathscr{V} .
$$

Из теоремы Брунна-Минковского (см. [24, теорема 21.1]) следует, что для любого $\bar{w} \in \mathscr{V}$ функция $f(r \bar{w})$ убывает при $r \geqslant 0$. Если $\mathscr{E}^{\prime}-$-ортогональная проекция $\mathscr{E}$ на $\mathscr{V}$, то мы имеем

$$
\begin{aligned}
\operatorname{Vol}_{n}\left(\mathscr{W}_{0} \cap \eta \mathscr{E}\right) & =\int_{\bar{w} \in \eta \mathscr{E}^{\prime}} f(\bar{w}) d \operatorname{Vol}_{\rho}(\bar{w}) \\
& =\int_{\eta \bar{w} \in \eta \mathscr{E}^{\prime}} f(\eta \bar{w}) d \operatorname{Vol}_{\rho}(\eta \bar{w})=\eta^{-\rho} \int_{\bar{w} \in \mathscr{E}^{\prime}} f(\eta \bar{w}) d \operatorname{Vol}_{\rho}(\bar{w}) \\
& \geqslant \eta^{-\rho} \int_{\bar{w} \in \mathscr{E}^{\prime}} f(\bar{w}) d \operatorname{Vol}_{\rho}(\bar{w})=\eta^{-\rho} \operatorname{Vol}\left(\mathscr{W}_{0}\right),
\end{aligned}
$$

что завершает доказательство леммы.

Определим $\Omega_{0}$ из соотношения

$$
\operatorname{Vol}(\mathscr{W})=\frac{L^{n}}{\Omega_{0}}
$$

Используя только (5.5), (5.6), мы получаем тело $\mathscr{W}_{0}$ объема

$$
\operatorname{Vol}\left(\mathscr{W}_{0}\right)=\frac{L^{n}}{\Omega}, \quad \Omega=A_{1} \ldots A_{n}
$$

Если мы просто возьмем $\mathscr{L}_{0}=\mathscr{W} \cap \mathbb{Z}^{n}$, то $\left|\mathscr{L}_{0}\right|$ может быть меньше, чем $\operatorname{Vol}(\mathscr{W})$. С помошью теоремы Блихфельда (см. [25, гл. 3 , теорема 1]) можно увеличить $\left|\mathscr{L}_{0}\right|$, так как согласно ей существует такой вектор $\bar{w}_{0}$, что

$$
\left|\left(\mathscr{W}+\bar{w}_{0}\right) \cap \mathbb{Z}^{n}\right|>\operatorname{Vol}(\mathscr{W}) .
$$

Тогда для $\mathscr{L}_{0}$ из (5.1) получаем, что $\left|\mathscr{L}_{0}\right|>L^{n} / \Omega_{0}$. Мы можем брать не все эти точки, а только часть их, чтобы было

$$
\left|\mathscr{L}_{0}\right|=\left[\frac{L^{n}}{\Omega_{0}}\right]+1
$$

Если мы увеличим $\Omega_{0}$, то (5.10) будет означать, что мы берем меньше точек, чем это возможно. 
Отметим, что вектор $\bar{w}_{0}$ определен с точностью до слагаемого из $\mathbb{Z}^{n}$, так что мы можем выбрать его удовлетворяюшим условиям

$$
\bar{w}_{0} \in[-0.5,0.5]^{n} .
$$

Согласно лемме 3.2 прибавление $\bar{w}_{0}$ к $\mathscr{W}$ не изменяет значение константы $\vartheta$ из (5.8), но ухудшает константу $\vartheta_{0}^{*}$ на слагаемое, не превосходяшее числа $\varepsilon^{*}$, задаваемого условием

$$
\varepsilon^{*}=\max \frac{\left\{|\bar{w} \cdot \ln \bar{\alpha}|: \bar{w} \in[-0.5,0.5]^{n}\right\}}{L} .
$$

Положим также

$$
\vartheta^{*}=\vartheta_{0}^{*}+\varepsilon^{*}
$$

и введем для удобства параметры

$$
\begin{aligned}
& B_{1}=\max \frac{\{|\tilde{\nu}(\bar{w})|: \bar{w} \in \mathscr{W}\}}{M_{0}}, \\
& B_{0}=\max \frac{\left\{|\tilde{\nu}(\bar{w})|: \bar{w} \in[-0.5,0.5]^{n}\right\}}{M_{0}},
\end{aligned}
$$

так что для $\bar{l} \in \mathscr{L}_{0}$ мы получаем, что $|\tilde{\nu}(\bar{l})| \leqslant\left(B_{1}+B_{0}\right) M_{0}$.

\section{§6. Лемма Зигеля}

Введем понятие длины вектора с весовьми коэффициентами, так как соответствуюшая теорема в [20] была сформулирована и доказана именно в этих терминах. Пусть даны вектор

$$
\bar{a}^{\prime \prime}=\left(a_{1}, \ldots, a_{J}\right) \in \mathbb{K}^{J}
$$

и набор положительных чисел (весовых коэффициентов)

$$
\mathscr{Q}=\left\{q_{j \sigma}: 1 \leqslant j \leqslant J ; \sigma \geqslant 1\right\} .
$$

Здесь при $\sigma \nmid \infty$ будем брать $q_{j \sigma}=1$. Через $\mathscr{Q}^{\prime}$ обозначим множество обратных значений. Определим локальные и абсолютную длины вектора $\bar{a}^{\prime \prime}$ с весовыми коэффищиентами $\mathscr{Q}$ с помошью равенств

$$
\begin{gathered}
\left\|\bar{a}^{\prime \prime}\right\|_{\mathscr{Q}, \sigma}=\left(\sum_{j=1}^{J} \frac{\left|a_{j}\right|_{\sigma}^{2}}{q_{j \sigma}^{2}}\right)^{1 / 2}, \quad 1 \leqslant \sigma \leqslant D_{\mathbb{K}}, \\
\left\|\bar{a}^{\prime \prime}\right\|_{\mathscr{Q}, \sigma}=\max \left\{\frac{\left|a_{j}\right|_{\sigma}}{q_{j \sigma}}: 1 \leqslant j \leqslant J\right\}, \quad \sigma>D_{\mathbb{K}}, \\
\left\|\bar{a}^{\prime \prime}\right\|_{\mathscr{Q}}=\left(\prod_{\sigma=1}^{\infty}\left\|\bar{a}^{\prime \prime}\right\|_{\mathscr{Q}, \sigma}\right)^{1 / D_{\mathbb{K}}} .
\end{gathered}
$$


(Если мы хотим определить высоты, то в архимедовых нормированиях надо брать максимум вместо суммирования.)

Для векторов $\bar{a}^{\prime \prime}, \bar{p}^{\prime \prime} \in \mathbb{K}^{J}$ вьполняются неравенства

$$
\left|\bar{a}^{\prime \prime} \cdot \bar{p}^{\prime \prime}\right|_{\sigma} \leqslant\left\|\bar{a}^{\prime \prime}\right\|_{\mathscr{Q}^{\prime}, \sigma} \cdot\left\|\bar{p}^{\prime \prime}\right\|_{\mathscr{Q}, \sigma}
$$

ЛЕмма 6.1 [20, теорема 3]. Пусть дань векторь

$$
\bar{a}_{i}^{\prime \prime}=\left(a_{i 1}, \ldots, a_{i J}\right) \in \mathbb{K}^{J}, \quad i=1, \ldots, I<J
$$

и набор 2 весовых коэффичиентов (6.1). Положим

$$
q_{j}=\prod_{\sigma=1}^{\infty} q_{j \sigma}^{1 / D_{\mathbb{K}}}, \quad 1 \leqslant j \leqslant J, \quad q=\left(q_{1} \ldots q_{J}\right)^{1 / J}
$$

Тогда система линейных уравнений

$$
\bar{a}_{i}^{\prime \prime} \cdot \bar{p}^{\prime \prime}=0, \quad 1 \leqslant i \leqslant I,
$$

имеет ненулевое решение $\bar{p}^{\prime \prime}=\left(p_{1}, \ldots, p_{J}\right) \in \mathbb{K}^{J}$, удовлетворяющее условию

$$
\begin{gathered}
\left\|\bar{p}^{\prime \prime}\right\|_{\mathscr{Q}} \leqslant \gamma\left(q^{-J} \prod_{i=1}^{I} \max \left\{q,\left\|\bar{a}_{i}^{\prime \prime}\right\|_{\mathscr{Q}^{\prime}}\right\}\right)^{1 /(J-I)}, \\
\gamma=|\Delta(\mathbb{K})|^{1 /\left(2 D_{\mathbb{K}}\right)} \max \left\{1,0.5 J^{1 / 2}\right\}
\end{gathered}
$$

әде $\Delta(\mathbb{K})$ - дискриминант поля $\mathbb{K}$.

ЗАМЕЧАНИЕ 6.1. В [20] в выражении для $\gamma$ стояло просто $0.5 J^{1 / 2}$ с условием $J \geqslant 4$, но из доказательства ясно, что $\gamma$ можно взять, как в (6.4). В данной работе $J>4$.

Смысл введения весовых коэффициентов состоит в том, что составляющие решения $\bar{p}^{\prime \prime}$ при больших множителях будут маль, а большие числа будут стоять только при малых сомножителях, так что их вклад в оценку выражений будет мал. Это главное отличие от результата, полученного в [26].

Другое отличие от [26] состоит в том, что мы не требуем, чтобы система была полного ранга. Так что теоремы из [26] требуют аккуратного применения или должны быть слегка уточнены с помошью рассуждений, использованных в [20]. Здесь важно, что высоты линейных форм (без весовых коэффициентов) оцениваются сверху числами не меньше 1, кроме нулевых форм, для которых тоже надо брать оценку не меньше 1.

Ввиду леммы 6.1 нам нужна оценка для $|\Delta(\mathbb{K})|$. 
ЛЕмма 6.2. Пусть даны ненулевые алгебрачческие числа $\alpha_{0}, \ldots, \alpha_{n}$, $\mathbb{K}=\mathbb{Q}\left(\alpha_{0}, \ldots, \alpha_{n}\right), \quad D_{\mathbb{K}}=[\mathbb{K}: \mathbb{Q}]$. Положим $\mathbb{K}_{0}=\mathbb{Q}\left(\alpha_{0}\right), \quad D_{0}=\left[\mathbb{K}_{0}: \mathbb{Q}\right]$, $u \mathbb{K}_{j}=\mathbb{K}_{j-1}\left(\alpha_{j}\right), \quad D_{j}=\left[\mathbb{K}_{j}: \mathbb{K}_{j-1}\right], \quad 1 \leqslant j \leqslant n$. Тогдa

$$
\begin{aligned}
& \ln |\Delta(\mathbb{K})| \leqslant D_{\mathbb{K}} \ln \left(D_{\mathbb{K}}\right)+2 D_{\mathbb{K}} \sum_{j=0}^{n}\left(D_{j}-1\right) h\left(\alpha_{j}\right), \\
& \ln |\Delta(\mathbb{K})| \leqslant D_{\mathbb{K}} \ln \left(D_{\mathbb{K}}\right)+2 D_{\mathbb{K}}\left(D_{\mathbb{K}}-1\right) \max \left\{h\left(\alpha_{j}\right): 0 \leqslant j \leqslant n\right\} .
\end{aligned}
$$

ДокАЗАТЕЛЬСТво. Положим $\bar{D}^{\prime}=\left(D_{0}, \ldots, D_{n}\right)$. Очевидно, что $D_{\mathbb{K}}=$ $D_{0} \ldots D_{n}$, а базис поля $\mathbb{K}$ над $\mathbb{Q}$ может быть взят в виде набора чисел $\omega\left(\bar{d}^{\prime}\right)=\bar{\alpha}^{\prime} \bar{d}^{\prime}$, $\overline{0}^{\prime} \leqslant \bar{d}^{\prime}<\bar{D}^{\prime}$. Рассмотрим число

$$
\Delta=\operatorname{det}\left(\omega\left(\bar{d}^{\prime}\right)^{(\sigma)}\right)_{1 \leqslant \sigma \leqslant D_{\mathbb{K}} ; \overline{0}^{\prime} \leqslant \bar{d}^{\prime}<\bar{D}^{\prime}}
$$

Из неравенства Адамара получается оценка

$$
|\Delta| \leqslant D_{\mathbb{K}}^{D_{\mathbb{K}} / 2} \prod_{j=0}^{n} \prod_{\sigma=1}^{D_{\mathbb{K}}} \max \left\{1,\left|\alpha_{j}\right|_{\sigma}\right\}^{D_{j}-1} .
$$

При геометрическом изображении поля $\mathbb{K} \mathbb{Z}$-модуль $\mathscr{M}_{0}$, порожденньй числами $\omega\left(\bar{d}^{\prime}\right)$, становится $D_{\mathbb{K}}$-мерной решеткой объема $\operatorname{Vol}\left(\mathscr{M}_{0}\right)=|\Delta|$. Очевидно, $\mathscr{M}_{0}$ лежит в дробном идеале $\mathscr{M}_{1}$, порожденном числами $\omega\left(\bar{d}^{\prime}\right)$, поэтому $\operatorname{Vol}\left(\mathscr{M}_{1}\right) \leqslant \operatorname{Vol}\left(\mathscr{M}_{0}\right)$.

Пусть мы имеем разложение идеалов $\left(\alpha_{j}\right)=\mathfrak{a}_{j} / \mathfrak{b}_{j}, 0 \leqslant j \leqslant n$, с цельми взаимно простыми идеалами $\mathfrak{a}_{j}, \mathfrak{b}_{j}$. Тогда идеал $\mathscr{M}_{2}=\mathscr{M}_{1} \prod_{j=0}^{n} \mathfrak{b}_{j}^{D_{j}-1}$ будет цельм. Его объем с учетом (6.7) будет

$$
\begin{aligned}
\operatorname{Vol}\left(\mathscr{M}_{2}\right) & =\operatorname{Vol}\left(\mathscr{M}_{1}\right) \prod_{j=0}^{n} \operatorname{Norm}\left(\mathfrak{b}_{j}\right)^{D_{j}-1}=\operatorname{Vol}\left(\mathscr{M}_{1}\right) \prod_{j=0}^{n} \prod_{\sigma>D} \max \left\{1,\left|\alpha_{j}\right|_{\sigma}\right\}^{D_{j}-1} \\
& \leqslant D_{\mathbb{K}}^{D_{\mathbb{K}} / 2} \prod_{j=0}^{n} \prod_{\sigma=1}^{\infty} \max \left\{1,\left|\alpha_{j}\right|_{\sigma}\right\}^{D_{j}-1} \\
& =\exp \left(0.5 D_{\mathbb{K}} \ln D_{\mathbb{K}}+D_{\mathbb{K}} \sum_{j=0}^{n}\left(D_{j}-1\right) h\left(\alpha_{j}\right)\right) .
\end{aligned}
$$

Так как $\mathscr{M}_{2}$ - целый идеал, то $\mathscr{M}_{2} \subseteq \mathscr{M}=(1)$, и поэтому

$$
|\Delta(\mathbb{K})|^{1 / 2}=\operatorname{Vol}(\mathscr{M}) \leqslant \operatorname{Vol}\left(\mathscr{M}_{2}\right),
$$

откуда следует неравенство (6.5).

Последовательно используя очевидное неравенство

$$
\left(D_{1}-1\right)+\left(D_{2}-1\right) \leqslant\left(D_{1} D_{2}-1\right), \quad D_{1}, D_{2} \geqslant 1,
$$

можно упростить (6.5) и получить (6.6), что доказывает лемму.

Отметим, что если у нас нет $\alpha_{0}$, то можно взять $\alpha_{0}=1$.

Лемма 6.2 - это единственное место в статье, где встречается именно $D_{\mathbb{K}}$, а не $D=D_{\mathbb{K}} / \varkappa$. 
ЗАМЕчАнИЕ 6.2. Оценка (6.5) точнее оценки

$$
\ln |\Delta(\mathbb{K})| \leqslant n\left(D_{\mathbb{K}}-1\right) D_{\mathbb{K}} \ln 2+2\left(D_{\mathbb{K}}-1\right) \sum_{j=1}^{n} h\left(\alpha_{j}\right) \operatorname{deg} \alpha_{j}
$$

из [18, лемма 2].

\section{§7. Некоторые базисные многочлены}

В оценках важно, каким образом представлять многочлены $P(z)$. Заслугой Фельдмана является осознание требования того, что рассматриваемые многочлены не обязательно должны быть из $\mathbb{Z}[x]$. Желаемое условие - это целозначность, т.е. что они должны принимать целые значения при целых значениях аргумента. Фельдман стал систематически использовать базисные многочлены

$$
\Delta(z, 0)=1, \quad \Delta(z, k)=\frac{z(z+1) \ldots(z+k-1)}{k !}, \quad k=1,2, \ldots
$$

Позднее эта система многочленов была модифищирована. Мы будем брать многочлены, введенные в [27]. Пусть $H \in \mathbb{N}$, тогда

$$
\Delta(z, l, H)=(\Delta(z, H))^{\lambda} \Delta(z, h), \quad l=\lambda H+h, \quad 0 \leqslant h \leqslant H, \quad l \in \mathbb{N}_{0} .
$$

Очевидно, $\operatorname{deg} \Delta(z, l, H)=l$. Положим также для $\gamma \neq 0$

$$
\Delta(\gamma z, l, H, m)=\frac{\Delta^{(m)}(\gamma z, l, H)}{\gamma^{m} m !} .
$$

Лемма 7.1. Пусть $H, L_{0}, N \in \mathbb{N} ; \quad M_{0}, L_{-1} \in \mathbb{N}_{0} ; \quad L_{0}=L_{-1} H+H_{1}$, $0 \leqslant H_{1} \leqslant H, d_{0}$ - наименьший общий знаменатель чисел

$$
\Delta(N x, l, H, m), \quad x \in \mathbb{Z}, \quad 0 \leqslant l \leqslant L_{0}, \quad 0 \leqslant m \leqslant M_{0} .
$$

Тогда

$$
\begin{aligned}
& d_{0} \leqslant \exp \left(1.03883 H M_{0}\right), \\
& d_{0} \leqslant H !^{L_{-1}} H_{1} !\left(\frac{2.8361 e\left(M_{0}+L_{-1}+1\right)}{L_{0}}\right)^{L_{0}} .
\end{aligned}
$$

Если $z \in \mathbb{C}$, то с некоторыми коэффициентами $\delta(l, m)>0$ выполняется

$$
\begin{aligned}
& |\Delta(z, l, H, m)| \leqslant \delta(l, m)\left(e\left(1+\frac{|z|}{H}\right)\right)^{l}, \\
& \sum_{l=0}^{L_{0}} \sum_{m=0}^{M_{0}} \delta(l, m) \leqslant \exp \left(L_{-1}+2+\frac{H}{e}\right) .
\end{aligned}
$$


ДоКАЗАТЕЛЬСТво (см. [27]). Здесь мы только вставили множитель $N$, зависимость от которого очевидна.

У нас будет

$$
N=2^{S},
$$

где $S$ - из $\S 4$. Смысл введения множителя $N$ состоит в том, что в дальнейшем мы будем подставлять $z=x / 2$, a $N$ позволяет избежать дробных значений аргумента.

ЗАмЕчАниЕ 7.1. Мы не будем использовать оценку (7.1), так как в нашем случае (7.2) будет лучше. Все предшествуюшие авторы использовали оценки типа (7.1). Мы также не будем использовать неравенства $(7.3),(7.4)$, поскольку присутствие множителя $N$ требует более аккуратных оценок.

Здесь и далее мы будем использовать количественный вариант формулы Стирлинга

$$
N^{N} e^{-N}(2 \pi N)^{1 / 2}<N !<N^{N} e^{-N}(2 \pi N)^{1 / 2} e^{1 /(12 N)}, \quad N \in \mathbb{N} .
$$

ЛЕмма 7.2. В обозначениях леммы 7.1 мы имеем

$$
H !^{L_{-1}} H_{1} ! \leqslant H^{L_{0}} e^{-L_{0}}(2 \pi H)^{L_{0} /(2 H)} \exp \left(\frac{L_{0}}{12 H^{2}}\right) .
$$

ДоКАЗАТЕЛЬСТво. Если $H_{1}=0$, то (7.7) является простым следствием (7.6). Пусть теперь $H_{1} \geqslant 1$. Положим $x=H_{1} / H(1 / H \leqslant x \leqslant 1)$. Тогда, используя (7.6), можно выписать неравенство

$$
\begin{gathered}
H !^{L_{-1}} H_{1} ! \leqslant H^{L_{0}} e^{-L_{0}}(2 \pi H)^{L_{0} /(2 H)} \exp \left(\frac{L_{0}}{12 H^{2}}+f(x)\right), \\
f(x)=H x \ln (x)-\frac{x \ln (2 \pi H)}{2}+\frac{\ln (2 \pi H x)}{2}-\frac{x-\frac{1}{x}}{12 H} .
\end{gathered}
$$

Дифференцированием мы получаем, что

$$
f^{\prime \prime}(x)=\frac{24(H x)^{2}-12(H x)+4}{24 H x^{3}},
$$

и легко видеть, что при $H x \geqslant 1$ выполняется $f^{\prime \prime}(x) \geqslant 0$, т.е. $f(x)$ выпукла вниз и принимает максимальное значение при $x=1 / H$ или $x=1$. При $x=1$ мы имеем $f(1)=0$, значит, в этом случае утверждение леммы верно. При $x=1 / H$ мы имеем

$$
f\left(\frac{1}{H}\right)=-\ln (H)-\frac{\ln (2 \pi H)}{2 H}+\frac{\ln (2 \pi)}{2}+\frac{1}{12}-\frac{1}{12 H^{2}} .
$$

Простое вычисление показывает, что максимальное значение $f(1 / H)$ достигается при $H=1$ и равно нулю, что доказьвает лемму. 
Лемма 7.3. Пусть $\gamma \neq 0, H \in \mathbb{N}, H>1, z_{0} \geqslant \max \{|z|, H\}$. Тогдa

$$
\left|\frac{\Delta^{(\tau)}(\gamma z, l, H, m)}{\tau !}\right| \leqslant \delta(l, m, \tau)\left(e\left(1+\frac{|\gamma| z_{0}}{H}\right)\right)^{l}
$$

əде

$$
\sum_{l=0}^{\infty} \sum_{m+\tau \leqslant l} \delta(l, m, \tau) \leqslant\left(1-\frac{e}{\sqrt{2 \pi H}}\right)^{-1} e^{(H+1) / e} .
$$

ДокАЗАТЕЛЬСТво. Производная содержит $\left(\begin{array}{c}l \\ m+\tau\end{array}\right)$ слагаемых с множителем $\left(\begin{array}{c}m+\tau \\ \tau\end{array}\right)$. Числитель каждого слагаемого оценивается через

$$
|\gamma|^{\tau}\left(|\gamma| z_{0}+H\right)^{l-m-\tau}=\left(\frac{H}{e}\right)^{l}\left(e\left(1+\frac{|\gamma| z_{0}}{H}\right)\right)^{l}|\gamma|^{\tau}\left(|\gamma| z_{0}+H\right)^{-m-\tau}
$$

что дает нам

$$
\begin{gathered}
\sum_{m+\tau \leqslant l}\left(\begin{array}{c}
l \\
m+\tau
\end{array}\right)\left(\begin{array}{c}
m+\tau \\
\tau
\end{array}\right)\left(\frac{1}{|\gamma| z_{0}+H}\right)^{m}\left(\frac{|\gamma|}{|\gamma| z_{0}+H}\right)^{\tau} \\
=\left(1+\frac{|\gamma|+1}{|\gamma| z_{0}+H}\right)^{l} \leqslant\left(\frac{H+1}{H}\right)^{l} .
\end{gathered}
$$

Теперь, положив $l=\lambda H+h(0 \leqslant h<H)$ и использовав (7.6) и неравенство $(1+1 / H)^{H}<e$, получим

$$
\begin{aligned}
& \sum_{l=0}^{\infty} \sum_{m+\tau \leqslant l} \delta(l, m, \tau) \leqslant \sum_{\lambda=0}^{\infty} \sum_{h=0}^{H-1}\left(\frac{H}{e}\right)^{l} \frac{\left(\frac{H+1}{H}\right)^{l}}{H !^{\lambda} h !} \\
& \quad=\sum_{\lambda=0}^{\infty}\left(\frac{\left(\frac{H+1}{e}\right)^{H}}{H !}\right)^{\lambda} \sum_{h=0}^{H-1} \frac{\left(\frac{H+1}{e}\right)^{h}}{h !} \leqslant e^{(H+1) / e} \sum_{\lambda=0}^{\infty}\left(\frac{e}{\sqrt{2 \pi H}}\right)^{\lambda}
\end{aligned}
$$

откуда следует (7.9).

ЗАмЕчАниЕ 7.2. Если у нас есть некоторая оценка модуля многочлена $P(z)$ для значений $|z| \leqslant z_{0}$, а мы хотим оценивать его для больших значений $z$, мы должны добавить множитель $\left(|z| / z_{0}\right)^{\operatorname{deg} P}$. 


\section{§ 8. Построение исходной вспомогательной функции}

Многочлены вспомогательной функции будем записывать в виде

$$
P_{0}(z, \bar{l})=\sum_{l_{0}=0}^{L_{0}} p_{l_{0}, \bar{l}} \Delta\left(N z, l_{0}, H_{0}\right), \quad \bar{l} \in \mathscr{L}_{0}
$$

Введем далее для краткости мульти-индекс

$$
\bar{l}^{\prime}=\left(l_{0}, \bar{l}\right) \in \mathscr{L}_{s}^{\prime}, \quad \mathscr{L}_{s}^{\prime}=\left\{0, \ldots, L_{0}\right\} \times \mathscr{L}_{s}
$$

Следующее важное соображение доказательства, характерное для однородного рационального случая, заключается в рассмотрении несколько иных вспомогательных функций, отличных от $G_{s}\left(z, \widetilde{m}^{\prime}\right), F_{s}\left(z, \widetilde{m}^{\prime}\right)$ из $(4.1),(4.2)$. Сначала мы можем, деля на подходящее число, заменить $\tilde{\chi}(\bar{l})$ на $\tilde{\nu}(\bar{l})$ (не в показателе). Это очевидное улучшение не сделано до сих пор. Не столь очевидное улучшение состоит в замене $\tilde{\nu}^{\widetilde{m}}(\bar{l})$ на $\Delta(\tilde{\nu}(\bar{l}), \widetilde{m})$. Возможность такой замены отличает форму (1.2) от формы (1.1) и позволяет получить оценку (1.4) вместо (1.3). Эта идея принадлежит Фельдману [28]. Так мы получаем новые функции (соответственно вместо (4.1), (4.2)):

$$
\begin{aligned}
& \Psi_{0}\left(z, \widetilde{m}^{\prime}\right)=\sum^{(0)}\left(N^{m_{0}} m_{0} !\right)^{-1} P_{0}^{\left(m_{0}\right)}(z, \bar{l}) \Delta(\tilde{\nu}(\bar{l}), \widetilde{m}) \tilde{\alpha}^{\tilde{\chi}(\bar{l}) z / b_{n}} \\
& \Phi_{0}\left(x, \widetilde{m}^{\prime}\right)=\sum^{(0)}\left(N^{m_{0}} m_{0} !\right)^{-1} P_{0}^{\left(m_{0}\right)}(x, \bar{l}) \Delta(\tilde{\nu}(\bar{l}), \widetilde{m}) \bar{\alpha}^{\bar{l} x}
\end{aligned}
$$

(в этом параграфе нам нужно только $s=0$ ). Используя лемму 3.1, мы можем преобразовывать функции (8.2), (8.3) в функции (4.1), (4.2) и обратно. Значит, система уравнений (4.3) эквивалентна системе

$$
\Phi_{0}\left(x, \widetilde{m}^{\prime}\right)=0, \quad\left|\widetilde{m}^{\prime}\right| \leqslant M_{0}, \quad x \in \mathscr{X}_{0} .
$$

Ввиду формул (8.1) мы можем рассматривать функции $\Phi_{0}\left(x, \widetilde{m}^{\prime}\right)$ как линейные формы от переменных $p_{\bar{l}^{\prime}} \in \mathbb{K}$. Условия (8.4) являются линейными уравнениями относительно $p_{\bar{l}^{\prime}}$. Чтобы их выполнить, применим лемму 6.1. Мульти-индекс $\bar{l}^{\prime}$ соответствует индексу $j$. Мульти-индекс $\left(x, \widetilde{m}^{\prime}\right)$ соответствует индексу линейной формы $i$. Весовые коэффициенты (6.1) выберем следуюшим образом:

$$
q_{\overline{l^{\prime}}, \sigma}=q_{\bar{l}^{\prime}}=\exp \left(c_{0}\left(L_{0}-l_{0}\right)\right), \quad 1 \leqslant \sigma \leqslant D, \quad \bar{l}^{\prime} \in \mathscr{L}_{0}^{\prime},
$$


с некоторой константой $c_{0}>0$. Теперь оценим параметры леммы 6.1. Принимая во внимание (5.10), мы имеем

$$
J=\left|\mathscr{L}_{0}^{\prime}\right|=\left(L_{0}+1\right)\left|\mathscr{L}_{0}\right|>\frac{\left(L_{0}+1\right) L^{n}}{\Omega_{0}} .
$$

Параметр $q$ из (6.3) равен

$$
q=\exp \left(\sum_{l_{0}=0}^{L_{0}} \sum^{(0)} c_{0} \frac{L_{0}-l_{0}}{J}\right)=\exp \left(c_{0} \sum_{l_{0}=0}^{L_{0}} \frac{L_{0}-l_{0}}{L_{0}+1}\right)=e^{c_{0} L_{0} / 2} .
$$

Количество уравнений равно

$$
\begin{gathered}
I=\left|\mathscr{X}_{0}\right| \Delta\left(M_{0}+1, n\right) \leqslant\left(2 X_{0}+1\right)\left(1+\varepsilon_{1}\right) \frac{M_{0}^{n}}{n !}, \\
\left(1+\varepsilon_{1}\right) \geqslant\left(1+\frac{n+1}{2 M_{0}}\right)^{n}
\end{gathered}
$$

(здесь и далее для оценки малых выражений мы будем вводить малые параметры $\varepsilon$, численное выражение для которых будет определено в конще доказательства, когда будет ясна величина главных членов).

Длины выражений $\Phi_{0}\left(x, \widetilde{m}^{\prime}\right)$, рассматриваемых как линейные формы от $p_{\bar{l}^{\prime}} \mathrm{c}$ весовыми коэффициентами из (8.5), оценим следуюшим образом. Оценки должны содержать компоненты:

1) для показательной части $\bar{\alpha}^{\bar{l} x}$,

$2)$ для модуля полиномиальной части $\Delta(N x, l, H, m)$,

3) для знаменателя $d_{0}$ многочленов,

4 ) для модуля $\Delta(\tilde{\nu}(\bar{l}), \widetilde{m})$ (это выражение возникает при дифференцировании вспомогательной функции, так что мы назовем его дифференциальной частью).

Вклад коэффициентов $\bar{p}^{\prime}$ в оценку $\Phi_{0}\left(x, \widetilde{m}^{\prime}\right)$ будет (вследствие выбора весовых коэффициентов) мал. Идея оптимального выбора параметров состоит в том, чтобы сделать оценки всех компонент одного порядка. Некоторое усиление может быть получено, если принять во внимание, что при экстраполяции роль этих компонент становится различной. В данной работе мы будем рассматривать отдельно показательную часть, а остальные будем выравнивать.

Множитель $\bar{\alpha}^{\bar{l} x}$ оценивается с учетом обозначений $\S 3$ и определения $\vartheta$ из (5.8) следуюшим образом:

$$
\exp \left(|x| h\left(\bar{\alpha}, \mathscr{L}_{0}\right)\right) \leqslant \exp (|x| h(\bar{\alpha}, \mathscr{W})) \leqslant \exp (|x| \vartheta n L) .
$$

Модуль $\Delta(\tilde{\nu}(\bar{l}), \widetilde{m})$ в соответствии с $(3.2),(5.13)$ оценивается как

$$
|\Delta(\tilde{\nu}(\bar{l}), \widetilde{m})| \leqslant \frac{\left(|\widetilde{m}|+\left(B_{1}+B_{0}\right) M_{0}\right)^{|\widetilde{m}|}}{|\widetilde{m}| !} \leqslant \frac{\left(\left(1+B_{1}+B_{0}\right) M_{0}\right)^{M_{0}}}{M_{0} !} .
$$


Если мы введем параметр $W$, удовлетворяющий условию

$$
W \geqslant \ln \left(e\left(1+B_{1}+B_{0}\right)\right),
$$

то в силу формулы Стирлинга (7.6) мы имеем неравенство

$$
|\Delta(\tilde{\nu}(\bar{l}), \widetilde{m})| \leqslant \frac{\exp \left(W M_{0}\right)}{\left(2 \pi M_{0}\right)^{1 / 2}}, \quad|\widetilde{m}| \leqslant M_{0}
$$

Знаменатель (с учетом (7.2), (7.7)) оценивается как

$$
d_{0} \leqslant \exp \left(c_{0} L_{0}\left(1+\varepsilon_{2}\right)\right)
$$

с некоторым $\varepsilon_{2}$, если мы возьмем $c_{0}$, удовлетворяюшее условию

$$
c_{0} \geqslant \ln \left(\frac{2.8361 H_{0}\left(M_{0}+L_{-1}+1\right)}{L_{0}}\right) .
$$

Если, кроме того, мы возьмем $c_{0}$, удовлетворяющее неравенству

$$
c_{0} \geqslant \ln \left(e\left(1+\frac{N\left(X_{0} 2^{n+1}+0.5\right)}{H_{0}}\right)\right),
$$

то из неравенств $(7.8),(8.5)$ мы получаем

$$
\begin{gathered}
\left|\Delta\left(N x, l_{0}, H_{0}, m_{0}\right)\right| q_{\bar{l}^{\prime}} \leqslant \delta\left(l_{0}, m_{0}, 0\right) \exp \left(c_{0} L_{0}\right), \\
|x| \leqslant Z_{0}+0.5
\end{gathered}
$$

ЗАмЕчАниЕ 8.1. В этом параграфе мы будем использовать (8.15) только для $|x| \leqslant X_{0}$, что может быть сделано, если $Z_{0} \geqslant X_{0}$. На самом деле $Z_{0}$ будет выбрано намного большим, чем $X_{0}$, чтобы упростить рассуждения в последуюших параграфах.

Мы видим, что полиномиальная часть оценивается так же, как и знаменатель. Если мы обеспечим $c_{0} L_{0}=W M_{0}$, то и дифференциальная часть будет оценена подобно полиномиальной части и знаменателю (cp. (8.11) с (8.12) и (8.13)).

Оставшуюся часть мы ввиду (7.9) оценим как

$$
\sum_{l_{0}=0}^{L_{0}} \delta\left(l_{0}, m_{0}, 0\right) \frac{\left|\mathscr{L}_{0}\right|^{1 / 2}}{\left(2 \pi M_{0}\right)^{1 / 2}} \leqslant 0.2 e^{\varepsilon_{3} c_{0} L_{0}} .
$$


Так что, собирая (8.6), (8.11), (8.12), (8.15), (8.16), в итоге мы получаем

$$
\left\|\Phi_{0}\left(x, \widetilde{m}^{\prime}\right)\right\|_{\mathscr{Q}^{\prime}} \leqslant 0.2 \exp \left(c_{0} L_{0}\left(3+\varepsilon_{2}+\varepsilon_{3}\right)+\vartheta|x| n L\right)
$$

(отметим, что правая часть больше, чем $q$ из (8.5), что важно в (6.4)). Перемножая неравенства (8.17), мы получаем

$$
\begin{aligned}
& \prod_{i=1}^{I} \max \left\{q,\left\|\bar{a}_{i}^{\prime \prime}\right\|_{\mathcal{Q}^{\prime}}\right\}=\prod_{x=-X_{0}} \prod_{\left|\tilde{m}^{\prime}\right| \leqslant M_{0}}\left\|\Phi_{0}\left(x, \tilde{m}^{\prime}\right)\right\|_{\mathscr{Q}^{\prime}} \\
& \leqslant \exp \left(I\left(c_{0} L_{0}\left(3+\varepsilon_{2}+\varepsilon_{3}\right)+\frac{\vartheta n L X_{0}\left(X_{0}+1\right)}{2 X_{0}+1}\right)\right) .
\end{aligned}
$$

Если мы обеспечим выполнение условия

$$
\frac{J c_{0} L_{0}}{2} \geqslant I\left(c_{0} L_{0}\left(3+\varepsilon_{2}+\varepsilon_{3}\right)+\frac{\vartheta n L X_{0}\left(X_{0}+1\right)}{2 X_{0}+1}\right)
$$

то ввиду $(8.5),(8.18)$ в соответствии с леммой 6.1 существует ненулевой вектор $\bar{p}^{\prime \prime}=\left\{p_{\bar{l}^{\prime}} \in \mathbb{K}: \bar{l}^{\prime} \in \mathscr{L}_{0}^{\prime}\right\}$, удовлетворяющий уравнениям (8.4) и условию (6.4), имеющему здесь вид

$$
\left\|\bar{p}^{\prime \prime}\right\|_{\mathscr{Q}} \leqslant \gamma \leqslant \exp \left(\varepsilon_{4} c_{0} L_{0}\right)
$$

Покажем, как выбрать значения параметров, удовлетворяюшие всем вышеприведенным условиям. Они будут выражены в терминах двух новых параметров $c_{1}, c_{2}$, характеризуюших соответственно отношения $M_{0} / L$ и $X_{0} / W$, значения которых будут заданы позже. Положим

$$
\begin{gathered}
X_{0}=c_{2} D W \geqslant \frac{1}{\varepsilon_{5}}, \quad \omega_{0}=\Omega_{0}\left(\frac{c_{1} \vartheta D}{e}\right)^{n}, \\
M_{0}=\frac{c_{0} L_{0}}{W}, \quad L=\frac{M_{0}}{c_{1} n \vartheta D}, \\
L_{0}+1 \geqslant 2 W D \omega_{0}\left(1+\varepsilon_{1}\right)\left(\left(2+\varepsilon_{5}\right)\left(3+\varepsilon_{2}+\varepsilon_{3}\right)+\left(1+\varepsilon_{5}\right) \frac{c_{2}}{c_{1}}\right) c_{2} e^{n} \frac{n^{n}}{n !} .
\end{gathered}
$$

Условие для $L_{0}$ можно получить, если подставить выражения для $M_{0}, L, X_{0}$ в (8.19) с учетом (8.6), (8.8). Таким образом мы выполним (8.19). Отметим, что у нас $W M_{0}=c_{0} L_{0}$.

Кроме этого мы должны обеспечить то, чтобы $M_{0}, L_{0}, X_{0} \in \mathbb{Z}$. Это может быть сделано, поскольку мы еше не зафиксировали параметры $c_{0}, W, L_{0}$. Сперва мы сделаем $X_{0} \in \mathbb{Z}$, немного увеличив $W$, затем добьемся, чтобы было $L_{0} \in \mathbb{Z}$, и, наконец, обеспечим $M_{0} \in \mathbb{Z}$ с помошью $c_{0}$. Параметр $H_{0}$ будет выбран так, чтобы правые части условий (8.13) и (8.14) были одного порядка. 


\section{§9. Интерполяционная формула}

Основной аналитический инструмент в методе Гельфонда-Бейкера состоит в применении интерполяционной формулы, получаемой из теоремы Коши о вычетах, которую мы здесь напомним. Мы также докажем некоторые связанные с ней технические неравенства, улучшаюшие ранее применявшиеся оценки.

Обозначим через $\mathscr{D}[x, R)$ и $\mathscr{D}[x, R]$ соответственно открытый и замкнутый круг в $\mathbb{C}$ с центром в $x$ и радиусом $R, \Gamma(x, R)$ обозначает окружность радиуса $R$ с центром в $x$.

Пусть дано конечное множество $\mathscr{X} \subseteq \mathbb{C}$ (узлов интерполяции) и для каждого $x \in \mathscr{X}$ - некоторое положительное целое $t=t(x)$ (кратность $x$ ). Обозначим такой объект через $(\mathscr{X}, \mathscr{T})$. Положим

$$
Q(z)=\prod_{x \in \mathscr{X}}(z-x)^{t(x)} .
$$

Этот многочлен определяет $(\mathscr{X}, \mathscr{T})$ и наоборот. Будем говорить, что

$$
Q(z)=\prod_{\tau=1}^{T} q_{\tau}(z), \quad q_{\tau}(z)=\prod_{t(x) \geqslant \tau}(z-x), \quad 1 \leqslant \tau \leqslant T,
$$

- стандартное разложение $Q(z)$. Зафиксируем также обозначения

$$
U=\operatorname{deg} Q(z), \quad u_{\tau}=\operatorname{deg} q_{\tau}(z) \geqslant 1, \quad 1 \leqslant \tau \leqslant T .
$$

Если $f(z)$ - аналитическая функция в $\mathscr{D}[0, R], \mathscr{X} \subseteq \mathscr{D}[0, R)$, то для $z \in \mathscr{D}[0, R) \backslash \mathscr{X}$ имеем

$$
f(z)=f_{1}(z)+f_{2}(z)
$$

где

$$
f_{1}(z)=\frac{1}{2 \pi i} \int_{\Gamma(0, R)} \frac{Q(z)}{Q(\zeta)} \cdot \frac{f(\zeta) d \zeta}{\zeta-z}
$$

и $\Gamma(0, R)$ обходится против часовой стрелки,

$$
f_{2}(z)=\sum_{x \in \mathscr{X}} \sum_{\tau=0}^{t(x)-1} \frac{f^{(\tau)}(x)}{2 \pi i \tau !} \int_{\Gamma(x, r)} \frac{Q(z)}{Q(\zeta)} \cdot \frac{(\zeta-x)^{\tau} d \zeta}{\zeta-z},
$$

$\Gamma(x, r)$ обходится по часовой стрелке и $z \notin \mathscr{D}[x, r] \subseteq \mathscr{D}[0, R), x \in \mathscr{X}$. Зададим также числа $r, r_{0}, \delta_{0}$ по формулам:

$$
\begin{aligned}
r_{0} & =\min \{|z-x|: x \in \mathscr{X}\}, \\
\delta_{0} & = \begin{cases}r_{0}, & \text { если }|\mathscr{X}|=1, \\
\min \{|x-y|: x, y \in \mathscr{X}, x \neq y\} & \text { иначе },\end{cases} \\
r & =\frac{\min \left\{r_{0}, \delta_{0}\right\}}{2} .
\end{aligned}
$$


Лемма 9.1. Пусть даны вышеописанные наборы $(\mathscr{X}, \mathscr{T}), \quad \mathscr{X} \subseteq \mathbb{R}, z \notin \mathscr{X}$, $z \in \mathbb{R}$. Стандартное разложение (9.1) $Q(\zeta)$ удовлетворяет (9.2), и каждый множитель $q_{\tau}(\zeta)$ является четным или нечетным с корнями, образующими арифметическую прогрессию с разностью $\delta_{\tau}>0$ (если $u_{\tau}=1$, то положим $\left.\delta_{\tau}=|z|\right)$. Положим также

$$
z_{\tau}=\max \left\{|z|, \frac{\delta_{\tau} u_{\tau}}{2}\right\}, \quad 1 \leqslant \tau \leqslant T, \quad z_{0} \geqslant \max \left\{z_{\tau}: 1 \leqslant \tau \leqslant T\right\}
$$

Тогда для каждого $\zeta \in \mathbb{C} c|\zeta|>z_{0}$ выполняется

$$
\left|\frac{Q(z)}{Q(\zeta)}\right| \leqslant\left(\frac{z_{0}}{|\zeta|}\right)^{U}
$$

и для кажддого $\zeta \in \Gamma(x, r), \quad x \in \mathscr{X}$ и г из (9.3) выполняется

$$
\left|\frac{q_{\tau}(z)}{q_{\tau}(\zeta)}\right| \leqslant\left(\frac{2 e z_{0}}{\delta_{0} u_{\tau}}\right)^{u_{\tau}}, \quad 1 \leqslant \tau \leqslant T
$$

ЗАмечАнИЕ 9.1. Такой многочлен $Q(\zeta)$ обычно получается, если множество $\mathscr{X}$ симметрично и образует арифметическую прогрессию, а также $t(-x)=t(x)$ и $t(x)$ убьвают при $x>0$. Здесь все $\delta_{\tau}$ равны. Однако в дальнейшем мы встретим более сложную ситуацию.

ДокАЗАТЕЛЬСтво. Принимая во внимание структуру корней $q_{\tau}(\zeta)$, мы видим, что при сдвиге $z$ на $\delta_{\tau}$ от центра значение $\left|q_{\tau}(z)\right|$ возрастает. Так что $\left|q_{\tau}(z)\right| \leqslant$ $q_{\tau}\left(z_{\tau}\right) \leqslant q_{\tau}\left(z_{0}\right)$ и $|Q(z)| \leqslant Q\left(z_{0}\right)$. Утверждение $(9.4)$ тогда следует из неравенства

$$
\frac{\left|z_{0}^{2}-x^{2}\right|}{\left|\zeta^{2}-x^{2}\right|} \leqslant \frac{z_{0}^{2}-x^{2}}{|\zeta|^{2}-x^{2}} \leqslant\left(\frac{z_{0}}{|\zeta|}\right)^{2}
$$

При доказательстве (9.5) будем опускать индекс $\tau$ в $q_{\tau}(z), u_{\tau}, \delta_{\tau}$. Также считаем, что $z_{0}=z$. Через $y$ обозначим какой-либо корень $q(\zeta)$. Рассмотрим теперь два случая.

Случай 1. $r=\frac{|z-x|}{2}<\frac{\delta}{2}$. Здесь мы имеем:

а) $\left|\frac{z-y}{\zeta-y}\right|=2$, если $y=x$;

b) $\left|\frac{z-y}{\zeta-y}\right| \leqslant \frac{\delta+2 r}{\delta-r} \leqslant 4$ (это возможно, только если $y, z$ находятся по разные стороны от $x$ и $\left.|x-y|=\delta_{0}\right)$;

с) $\left|\frac{z-y}{\zeta-y}\right| \leqslant \frac{2 \delta+2 r}{2 \delta-r}<2$ для всех прочих $y$.

Если $u=1$, то (9.5) верно, так как всегда $z=z_{0} \geqslant \delta_{0}$. 
Если $u=2$, то (9.5) нетривиально только в случае b). Здесь $\pm y$ являются корнями $q(\zeta), \delta=2 y$ и (9.5) следует из неравенства

$$
\left|\frac{q(z)}{q(\zeta)}\right| \leqslant \frac{2(2 y+2 r)}{2 y-r} \leqslant\left(2 e \frac{\max \{y+2 r, 2 y\}}{4 y}\right)^{2},
$$

легко проверяемого отдельно для $0<r<y$ и $y \leqslant r \leqslant 2 y$ в соответствии с максимальным значением в определении $z_{\tau}$.

Если $u \geqslant 3$, то в этом случае мы имеем

$$
\left|\frac{q(z)}{q(\zeta)}\right| \leqslant 4 \cdot 2^{u-1}<e^{u} \leqslant\left(\frac{2 e z_{\tau}}{\delta u}\right)^{u} \leqslant\left(\frac{2 e z_{0}}{\delta_{0} u}\right)^{u} .
$$

Случай 2. $r=\delta_{0} / 2$. Здесь $|q(z)| \leqslant\left|q\left(z_{0}\right)\right| \leqslant z_{0}^{u}$. Так что для получения (9.5) остается проверить неравенство

$$
|q(\zeta)| \geqslant\left(\frac{\delta_{0} u}{2 e}\right)^{u}
$$

Точка $x$ может не быть нулем $q(\zeta)$, так что сначала мы, не увеличивая $|q(\zeta)|$, совместим $x$ с одним из корней. Сосчитаем число корней слева $\left(v_{1}\right)$ и справа $\left(v_{2}\right)$ от $x$. Если $v_{1}>v_{2}$, то совмешаем $x$ с ближайшим нулем $y$ слева, если $v_{1}<v_{2}$, то справа, если $v_{1}=v_{2}$, то совмешаем $x$ с ближайшим нулем к $\zeta$. При этом сдвигаем $\zeta$, не меняя $\operatorname{Im} \zeta$, до ближайшей точки на $\Gamma(y, r)$. После этого сожмем все нули так, что расстояние между ними станет $\delta_{0}$. Так что в оставшейся части доказательства можно считать $\delta=\delta_{\tau}=\delta_{0}$.

С учетом структуры корней многочлена $q(\zeta)$ мы можем сдвигать $\Gamma(x, r)$ вместе с $\zeta$ к центру на $\delta$. Это уменьшает $|q(\zeta)|$. Так что можно считать $x$ ближайшим к нулю. Следующее соображение основано на неравенствах

$$
|\zeta-y| \geqslant|y-x|-r, \quad|(\zeta-y-x)(\zeta-x+y)| \geqslant y^{2}-r^{2} .
$$

Для пар корней $q(\zeta)$, симметрично расположенных относительно $x$, мы применим второе из этих неравенств, а для остальных - первое. Это дает то, что минимум $|q(\zeta)|$ достигается для $\zeta \in \mathbb{R}$, ближайшего к нулю. Здесь имеются две возможности: когда $u$ четно $(u=2 X)$ и когда $u$ нечетно $(u=2 X+1)$. Для простоты далее примем $\delta=2$, что возможно в силу однородности обеих частей (9.6) для $\zeta \in \Gamma(x, \delta / 2)$.

Если $u=2 X+1$, то

$$
\begin{aligned}
|q(\zeta)| \geqslant|q(1)|=(2 X-1) ! !(2 X+1) ! !=\frac{((2 X+1) ! !)^{2}}{2 X+1}=\frac{\left(\frac{(2 X+1) !}{2^{X} X !}\right)^{2}}{2 X+1} \\
>\frac{\left(\frac{u^{u} e^{-u}}{2^{X} X^{X} e^{-X}}\right)^{2}}{2 X}=\left(\frac{u}{e}\right)^{u} \frac{\left(1+\frac{1}{2 X}\right)^{2 X+1}}{e}>\left(\frac{u}{e}\right)^{u} .
\end{aligned}
$$


Здесь мы использовали (7.6) для $N=2 X+1$ и $N=X$, а также неравенство $(1+1 / N)^{N+1}>e$.

Подобным же образом разбирается случай $u=2 X$. Мы имеем

$$
|q(\zeta)| \geqslant|q(0)|=((2 X-1) ! !)^{2}=\left(\frac{(2 X) !}{2^{X} X !}\right)^{2}>\left(\frac{u^{u} e^{-u}}{2^{X} X^{X} e^{-X}}\right)^{2}=\left(\frac{u}{e}\right)^{u}
$$

что доказывает (9.6) и лемму.

Следуюшее рассуждение мы будем использовать неоднократно, поэтому выделим его в виде леммы.

ЛЕмма 9.2. Пусть $f(z, \zeta)$ - функиия, определенная для некоторого данного $z \in \mathbb{R}$ и аналитическая по $\zeta$. Дань $(\mathscr{X}, \mathscr{T}), z \notin \mathscr{X}$, с соответствующим $Q(\zeta)$, удовлетворяющим условиям леммы $9.1 ; \delta_{0}, r, U, T-и з$ (9.1)-(9.3), $z_{0}-$ из леммы 9.1. Пусть $f(z, \zeta)$ удовлетворяет с некоторыми положительными $a_{0}, a_{1}, a_{2}, a_{3}, a_{4}$ и некоторым $z_{1} \geqslant|z|$ при $|\zeta| \leqslant R$ неравенству

$$
\begin{gathered}
|f(z, \zeta)| \leqslant a_{0} \exp \left(a_{1}+a_{2} z_{1}+a_{3} \ln \left(\frac{R}{z_{0}}\right)+a_{4} R\right), \\
R=\frac{U-a_{3}}{a_{4}} .
\end{gathered}
$$

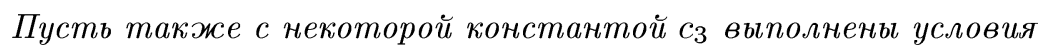

$$
\begin{gathered}
0<c_{3} \leqslant \ln \left(\frac{a_{1}+a_{2} z_{1}}{a_{4} z_{0} c_{3} e}\right), \\
U \geqslant a_{3}+\frac{a_{1}+a_{2} z_{1}}{c_{3}} .
\end{gathered}
$$

Тогда

$$
|f(z, z)|<2 a_{0}+\sum_{x \in \mathscr{X}} \sum_{\tau=0}^{t(x)-1} 2 r^{\tau}\left|\frac{Q(z)}{Q\left(\zeta_{x}\right)}\right| \cdot \frac{\left|f^{(\tau)}(z, x)\right|}{\tau !}
$$

(производные берутся по $\zeta, \zeta_{x} \in \Gamma(x, r)$ - точка с минимальныцм соответствующим значением $|Q(\zeta)|)$. 
ДоКАЗАТЕЛЬСТВО. Применим интерполящионную формулу для $f(z, \zeta)$ и $\zeta=z$, взяв для первого слагаемого оценку (9.4). В первом случае мы из (9.7), (9.8) имеем

$$
\begin{gathered}
R \geqslant \frac{a_{1}+a_{2} z_{1}}{c_{3} a_{4}} \geqslant \exp \left(c_{3}\right) e z_{0}>e z_{0}, \\
|\zeta-z| \geqslant R-z_{0} \geqslant\left(1-\frac{1}{e}\right) R>\frac{R}{2}, \\
\left|\frac{f(z, \zeta) Q(z)}{Q(\zeta)}\right|<a_{0} \exp \left(a_{1}+a_{2} z_{1}+a_{3} \ln \left(\frac{R}{z_{0}}\right)+a_{4} R-U \ln \left(\frac{R}{z_{0}}\right)\right) \\
=a_{0} \exp \left(a_{1}+a_{2} z_{1}-\left(U-a_{3}\right) \ln \left(\frac{U-a_{3}}{a_{4} z_{0} e}\right)\right) \\
\leqslant a_{0} \exp \left(c_{3}\left(U-a_{3}\right)-\left(U-a_{3}\right) \ln \left(\frac{a_{1}+a_{2} z_{1}}{c_{3} a_{4} z_{0} e}\right)\right) \\
\leqslant a_{0} \exp \left(\left(U-a_{3}\right)\left(c_{3}-\ln \left(\frac{a_{1}+a_{2} z_{1}}{c_{3} a_{4} z_{0} e}\right)\right) \leqslant a_{0},\right.
\end{gathered}
$$

что дает оценку первого слагаемого в правой части (9.10).

Второе слагаемое справа в (9.10) немедленно получается из интерполяционной формулы, что доказывает лемму. Множитель $\left|Q(z) / Q\left(\zeta_{x}\right)\right|$ будет далее оценен с помощью неравенства (9.5).

\section{§10. Индуктивный шаг доказательства}

Теперь введем набор функций, сходных с функциями (8.3):

$$
\begin{aligned}
\Phi_{s}\left(x, \widetilde{m}^{\prime}\right) & =\sum^{(s)}\left(\left(2^{-s} N\right)^{m_{0}} m_{0} !\right)^{-1} P_{s}^{\left(m_{0}\right)}(x, \bar{\lambda}) \Delta(\tilde{\nu}(\bar{l}), \widetilde{m}) \bar{\alpha}^{\bar{l} x / 2^{s}} \\
& =\gamma_{s}^{x} \sum^{(s)}\left(\left(2^{-s} N\right)^{m_{0}} m_{0} !\right)^{-1} P_{s}^{\left(m_{0}\right)}(x, \bar{\lambda}) \Delta(\tilde{\nu}(\bar{l}), \widetilde{m}) \bar{\alpha}^{\bar{\lambda} x}
\end{aligned}
$$

Здесь суммирование проводится по набору мульти-индексов $\bar{\lambda} \in \mathscr{L}_{s}, \bar{l}=\bar{l}(\bar{\lambda})$ определено с помошью (4.9), $\gamma_{s}=\bar{\alpha}^{\bar{\lambda}_{s} / 2^{s}}, \bar{\lambda}_{s}$ - также из (4.9). Для $s=0$ положим $\bar{\lambda}_{0}=\overline{0}$ и $\gamma_{0}=1$.

Индуктивньй шаг доказательства состоит из двух частей.

Часть 1. Прежде чем доказывать (4.5), установим, что

$$
\Phi_{s}\left(x, \widetilde{m}^{\prime}\right)=0, \quad x \in\left\{0, \pm 1, \pm 2, \ldots, \pm Z_{s}\right\}, \quad\left|\widetilde{m}^{\prime}\right| \leqslant M(x),
$$

с некоторыми $Z_{s}$ и кратностями $M(x)$, которые мы определим позже.

Часть 2. В ней мы докажем равенства

$$
\Phi_{s}\left(\frac{x}{2}, \widetilde{m}^{\prime}\right)=0, \quad x \in \mathscr{X}_{s+1}, \quad\left|\widetilde{m}^{\prime}\right| \leqslant M_{s+1}
$$


эквивалентные (4.5). Мы также зададим кратность $M_{s+1}$ несколько позже и проверим, что она согласуется с (4.6).

Положим в части $1 \mathbb{L}_{1}=\mathbb{K}$ и в части $2 \mathbb{L}_{2}=\mathbb{K}\left(\sqrt{\alpha_{1}}, \ldots, \sqrt{\alpha_{n}}\right)$. При этом видно, что при $z=x \in \mathbb{Z}$ в части $1(j=1)$ и при $z=x / 2, x \in \mathbb{Z}$, в части $2(j=2)$ значения $\left(\Phi_{s}\left(z, \widetilde{m}^{\prime}\right)\right)^{2^{s}}$ будут из поля $\mathbb{L}_{j}$.

Пронумеруем нормирования поля $\mathbb{L}_{j}$, как было описано в $\S 2$, индексами $\sigma$ и возьмем набор индексов $\mathscr{S}^{\prime}$, соответствующий вложениям $\mathbb{L}_{j}$ в $\mathbb{C}$, для которых $\mathbb{L}_{j}$ остается неподвижным или комплексно-сопряженным (если $\varkappa=2$ ), так что $\left|\mathscr{S}^{\prime}\right|=\varkappa$. Остальные индексы образуют набор $\mathscr{S}$. Отметим, что для $\sigma \in \mathscr{S}^{\prime}$ значения $\left|\Phi_{s}(x, \tilde{m})\right|_{\sigma}$ равны. Теперь введем еще функции

$$
\begin{gathered}
\Psi_{s}\left(\zeta, \widetilde{m}^{\prime}\right)=\sum^{(s)}\left(\left(2^{-s} N\right)^{m_{0}} m_{0} !\right)^{-1} P_{s}^{\left(m_{0}\right)}(\zeta, \bar{\lambda}) \Delta(\tilde{\nu}(\bar{l}), \tilde{m}) \tilde{\alpha}^{\tilde{\chi}(\bar{l}) \zeta /\left(2^{s} b_{n}\right)} \\
f(z, \zeta)=f_{j}(z, \zeta)=\Psi_{s}\left(\zeta, \widetilde{m}^{\prime}\right)\left(\prod_{\sigma \in \mathscr{S}}\left|\Phi_{s}\left(z, \tilde{m}^{\prime}\right)\right|_{\sigma}\right)^{1 / \varkappa}, \quad j=1,2 .
\end{gathered}
$$

Если предположить малость линейной формы от логарифмов, то значение $\Psi_{s}\left(z, \widetilde{m}^{\prime}\right)$ будет примерно равно $\Phi_{s}\left(z, \widetilde{m}^{\prime}\right)$, а значение $|f(z, z)|$ будет либо примерно равно 1 в соответствии с формулой произведения, либо равно 0. Так что нашей задачей будет показать, что $|f(z, z)|$ достаточно мало, чтобы исключить первый случай.

Разность между $\left|\Phi_{s}\left(\zeta, \widetilde{m}^{\prime}\right)\right|_{1}$ и $\left|\Psi_{s}\left(\zeta, \widetilde{m}^{\prime}\right)\right|$ может быть оценена с учетом определения $\tilde{\chi}(\bar{l})$ из $\S 3$ с помощью неравенства

$$
\begin{aligned}
& \left|\bar{\alpha}^{\bar{l} \zeta / 2^{s}}-\tilde{\alpha}^{\tilde{\chi}(\bar{l}) \zeta /\left(2^{s} b_{n}\right)}\right|=\left|\bar{\alpha}^{\bar{l} \zeta / 2^{s}}\right| \cdot\left|e^{-\Lambda \zeta l_{n} /\left(2^{s} b_{n}\right)}-1\right| \\
& \quad \leqslant \exp \left(\vartheta^{*} L\left|\frac{\zeta}{2^{s}}\right|\right) \cdot 1.1 \cdot\left|\frac{\Lambda \zeta l_{n}}{2^{s} b_{n}}\right|
\end{aligned}
$$

где $\Lambda$ - наша линейная форма (1.2), предполагаемая малой.

Здесь мы использовали определение (5.12) числа $\vartheta^{*},(5.7),(5.11)$ и то, что для $z \in \mathbb{C}$ c $|z| \leqslant 0.1$ выполняется $\left|e^{z}-1\right| \leqslant 1.1|z|$.

Оценка $|f(z, \zeta)|$ проводится так же, как и оценка $\left\|\Phi_{0}\left(z, \widetilde{m}^{\prime}\right)\right\|_{\mathscr{Q}^{\prime}}$, но с добавлением (8.20), с помощью (6.2) и с учетом того, что мы извлекаем корень не степени $\left[\mathbb{L}_{j}: \mathbb{Q}\right]$, а $\varkappa$. Так что соответствующая аналогичная оценка будет содержать в показателе дополнительньй множитель $\left[\mathbb{L}_{j}: \mathbb{Q}\right] / \varkappa$. Также предположим, что

$$
\left|\frac{\Lambda \zeta l_{n}}{2^{s} b_{n}}\right|<0.1
$$


для всех $\zeta$, которые будут встречаться в дальнейшем. Тогда мы имеем условие (9.7) леммы 9.2 , где

$$
\begin{gathered}
a_{0}=0.22, \quad a_{1}=a_{1 s j}=c_{0} L_{0}\left(3+\varepsilon_{2}+\varepsilon_{3}+\varepsilon_{4}\right) \frac{\left[\mathbb{L}_{j}: \mathbb{Q}\right]}{\varkappa}, \\
a_{2}=a_{2 s j}=\vartheta\left(2^{-s} L\right) n \frac{\left[\mathbb{L}_{j}: \mathbb{Q}\right]}{\varkappa}, \\
a_{3}=L_{0}, \quad a_{4}=a_{4 s}=\vartheta^{*} 2^{-s} L .
\end{gathered}
$$

Введем еще несколько констант:

$$
\begin{gathered}
\eta_{s}=\frac{a_{2 s 1}}{c_{3}}+\frac{\left(a_{3}+a_{1 s 1}\right) / c_{3}}{2 X_{s}}+\frac{1}{X_{s}}, \\
\xi_{s}=\frac{a_{2 s 1}}{2 c_{3}}, \quad \eta_{0 s}=\frac{a_{1 s 1}}{4 X_{s} c_{3}} .
\end{gathered}
$$

Будем доказывать равенства (10.1) индукцией по $|x|$. Основой служат равенства для $x \in \mathscr{X}_{s}$ и $M(x)=M_{s}$. Следующим шагом будет доказательство их для

$$
\begin{gathered}
x=z \in \mathbb{Z}, \quad|z| \leqslant 2 X_{s}, \quad z \notin \mathscr{X}_{s}, \quad z_{0}=z_{1}=2 X_{s}, \\
\left|\tilde{m}^{\prime}\right| \leqslant M(z)=\left[M_{s}-\eta_{s}\right]+1 .
\end{gathered}
$$

Различие между случаями $s=0$ и $s>0$ состоит в том, что $s=0$ нам надо доказать (10.1) для $X_{0}<|z| \leqslant 2 X_{0}$, а при $s>0$ нам надо доказать (10.1) для четных $z$ c $|z| \leqslant 2 X_{s}$. Теперь можно приступить к индуктивному доказательству (10.1) для

$$
\begin{gathered}
x=z \in \mathbb{Z}, \quad 2 X_{s}<|z| \leqslant Z_{s}, \quad z_{0}=z_{1}=|z| \\
\left|\widetilde{m}^{\prime}\right| \leqslant M(z)=\left[M_{s}-\eta_{s}-\xi_{s} \ln \left(\frac{|z|}{2 X_{s}}\right)\right]+1 \\
Z_{s}=2^{n+1} X_{s} .
\end{gathered}
$$

Отсюда видно, что кратность $M(z)$ четна и убывает при $z>2 X_{s}$. При использовании леммы 9.2 мы включим в множество узлов интерполяции $\mathscr{X}$ те $x \in \mathbb{Z}$, для которых либо $M(x)>M(z)$, либо $|x|<|z|$, если $M(x)=M(z)$. Кратности будут равны

$$
t(x)=M(x)-M(z)+1, \quad x \in \mathscr{X} .
$$

При доказательстве (10.2) (часть $2, j=2$ ) будем брать

$$
\mathscr{X}=\left\{0, \pm 1, \ldots, \pm Z_{s}\right\}
$$


так что для $z=x / 2, x \in \mathscr{X}_{s+1}$, с учетом того, что $X_{s+1}=2 X_{s}$, можно брать

$$
\begin{gathered}
|z| \leqslant z_{1}=2 X_{s}-1, \quad z_{0}=Z_{s}+0.5, \\
M_{s+1}=\left[M_{s}-\eta_{s}-\xi_{s} n \ln 2-\eta_{0 s}\right]+1, \\
t(x)=M(x)-M_{s+1}+1 .
\end{gathered}
$$

Отметим, что в сравнении с наибольшим $x$ из части 1 мы добавили к $\mathscr{X}$ две точки $\pm Z_{s}$ и немного увеличили кратности.

Идея включения всех ранее полученных точек с доказанньми для них кратностями была предложена автору А. Шмелевым, но пересмотреть должным образом технику экстраполирования оказалось технически сложной задачей.

Будем брать стандартное разложение многочлена $Q(\zeta)$, соответствующее вьшеописанному набору $(\mathscr{X}, \mathscr{T})$. Следующие леммы позволяют оценивать $U=\operatorname{deg} Q(\zeta)$ и другие параметры из $§ 9$.

Лемма 10.1. Пусть $\varphi(z)$ - выпуклая убывающая функщия, $X^{\prime}, X^{\prime \prime}, Y^{\prime}$ принадлежат $\mathbb{Z}$ и удовлетворяют условиям: $X^{\prime}<X^{\prime \prime}, Y^{\prime} \leqslant \varphi\left(X^{\prime \prime}\right)$. Положим $\mathscr{E}=\left\{(x, y) \in \mathbb{R}^{2}: X^{\prime}<x \leqslant X^{\prime \prime}, Y^{\prime} \leqslant y \leqslant \varphi(x)\right\}$. Тогда

$$
\operatorname{Card}\left(\mathscr{E} \cap \mathbb{Z}^{2}\right) \geqslant \operatorname{Vol}(\mathscr{E})-\frac{\varphi\left(X^{\prime}\right)-\varphi\left(X^{\prime \prime}\right)}{2} .
$$

ЗАмЕЧАнИЕ 10.1. Если $\varphi(x)$ не выпукла, то разность в оценке не надо делить на 2 . Если $\varphi(x)$ возрастает, то в условиях надо взять $Y^{\prime} \leqslant \varphi\left(X^{\prime}\right), X^{\prime} \leqslant x<X^{\prime \prime}$ и в оценке переставить $X^{\prime}$ и $X^{\prime \prime}$.

ДокАЗАТЕЛЬСТво. Определим для целых $m, n$ множества

$$
\begin{gathered}
\mathscr{S}_{m n}=\left\{(x, y) \in \mathbb{R}^{2}: m-1<x \leqslant m, n \leqslant y<n+1\right\}, \quad \mathscr{S}_{m}=\bigcup_{n} \mathscr{S}_{m n}, \\
\mathscr{E}^{\prime}=\bigcup_{(m, n) \in \mathscr{E}} \mathscr{S}_{m n}, \quad \mathscr{E}_{m}=\left(\mathscr{E} / \mathscr{E}^{\prime}\right) \cap \mathscr{S}_{m} .
\end{gathered}
$$

Из свойств функции $\varphi(z)$ мы получаем, что все множества $\mathscr{E}_{m}$ либо пусты, либо являются криволинейными прямоугольными треугольниками с прямыми катетами и выпуклой вниз гипотенузой. Их горизонтальные катеты меньше 1 , а сумма вертикальных катетов не больше, чем $\left(\varphi\left(X^{\prime}\right)-\varphi\left(X^{\prime \prime}\right)\right)$. Отсюда получаем

$$
\begin{aligned}
\operatorname{Vol}(\mathscr{E})-\operatorname{Card}\left(\mathscr{E} \cap \mathbb{Z}^{2}\right) & =\operatorname{Vol}(\mathscr{E})-\operatorname{Vol}\left(\mathscr{E}^{\prime}\right) \leqslant \operatorname{Vol}\left(\mathscr{E} / \mathscr{E}^{\prime}\right) \\
& =\sum_{m} \operatorname{Vol}\left(\mathscr{E}_{m}\right) \leqslant \frac{\varphi\left(X^{\prime}\right)-\varphi\left(X^{\prime \prime}\right)}{2} .
\end{aligned}
$$


Лемма 10.2. Для вышеописанного набора $(\mathscr{X}, \mathscr{T})$ мы имеем $r=1 / 2 ;$ в части $1(j=1) \delta_{0}=2$ для $\mathscr{X}=\mathscr{X}_{s} \quad(s>0)$, а иначе $\delta_{0}=1 ;$ в части $2(j=2)$ $\delta_{0}=1 ;$ в обоих случаях $(j=1,2)$ для $U$ выполняется $(9.9)$ с $a_{0}, a_{1}, a_{2}, a_{3}$ из (10.5).

ДокАЗАТЕЛЬСТво. Оценки для $\delta_{0}, r$ очевидны. Для $|z| \leqslant 2 X_{s}$ мы просто имеем $U \geqslant 2 X_{s} \eta_{s}$ и (9.9) выполнено. Так что далее мы будем считать, что $|z|>2 X_{s}$. Будем использовать лемму $10.1 \mathrm{c}$

$$
\begin{gathered}
y=\varphi(x)=M_{s}-\eta_{s}-\xi_{s} \ln \left(\frac{x}{2 X_{s}}\right)+1 \\
X^{\prime}=2 X_{s}, \quad X^{\prime \prime}=z_{0}, \quad Y^{\prime}=\left[\varphi\left(X^{\prime \prime}\right)\right] .
\end{gathered}
$$

Все условия этой леммы выполнены. Интегрируя, мы получаем, что для множества $\mathscr{E}$ из этой леммы выполняется

$$
\operatorname{Vol}(\mathscr{E}) \geqslant \xi_{s}\left(z_{0}-2 X_{s}\right)-2 X_{s}\left(\varphi\left(X^{\prime}\right)-\varphi\left(X^{\prime \prime}\right)\right)
$$

Здесь для интегрирования удобнее брать обратную к $\varphi(x)$ функцию

$$
x=\varphi^{-1}(y)=2 X_{s} \exp \left(\frac{M_{s}-\eta_{s}-y+1}{\xi_{s}}\right)
$$

Для оценки $U$ мы можем сосчитать точки $(x, y) \in \mathbb{Z}^{2}$, удовлетворяющие неравенствам $|x|<z_{0}, M\left(z_{0}\right) \leqslant y \leqslant M(x)$. Используя лемму 10.1, мы должны принять во внимание симметрию $M(x)$, а также то, что надо исключить две точки с $x= \pm z_{0}$. Все точки мы сгруппируем в соответствии со значением $x: x=0$, $0<|x| \leqslant X_{s}, X_{s}<|x| \leqslant 2 X_{s}, 2 X_{s}<|x| \leqslant z_{0}$. Это дает

$$
\begin{aligned}
U \geqslant & \left(\varphi\left(X^{\prime}\right)-\varphi\left(X^{\prime \prime}\right)\right)+2 X_{s}\left(M_{s}-\left[\varphi\left(X^{\prime \prime}\right)\right]+1\right)+2 X_{s}\left(\left[\varphi\left(X^{\prime}\right)\right]-\left[\varphi\left(X^{\prime \prime}\right)\right]+1\right) \\
& +2\left(\xi_{s}\left(z_{0}-2 X_{s}\right)-2 X_{s}\left(\varphi\left(X^{\prime}\right)-\varphi\left(X^{\prime \prime}\right)\right)-\left(\varphi\left(X^{\prime}\right)-\frac{\varphi\left(X^{\prime \prime}\right)}{2}-1\right)\right) \\
\geqslant & 2 \xi_{s} z_{0}+2 X_{s}\left(M_{s}+1-\varphi\left(X^{\prime}\right)-2 \xi_{s}\right)-2 .
\end{aligned}
$$

Принимая во внимание (10.7), мы получаем (9.9) для $j=1$.

Оценка $U$ в части 2 будет такая же, как в части 1 для $x=Z_{s}$, но с добавлением слагаемого не меньше, чем $2 Z_{s} \eta_{0 s}$ (мы снова используем лемму 10.1 с другим $Y^{\prime}$ ). Значит,

$$
U \geqslant a_{3}+\frac{a_{1 s 1}+a_{2 s 1} Z_{s}}{c_{3}}+2 \geqslant a_{3}+\frac{a_{1 s 2}+a_{2 s 2} X_{s+1}}{c_{3}}+\frac{a_{1 s 1}}{c_{3}} .
$$


Поскольку теперь $z_{1}=X_{s+1}$, то мы имеем (9.9) для $j=2$, что доказывает леммy 10.2 .

В силу выбора параметров (10.5)-(10.10) лемма 10.2 дает, что условия (9.7), (9.9) леммы 9.2 выполнены. Условие (9.8) будет обсуждаться в следующем параграфе. Здесь же мы только примем то, что оно тоже вьполнено. Таким образом, мы имеем, что первое слагаемое в $f(z, z)$ меньше, чем 0.44. Если мы докажем, что второе слагаемое тоже меньше 0.44 , то получим, что $|f(z, z)| \leqslant 0.88$ и в предположении о малости линейной формы от логарифмов - что $\Phi_{s}\left(x, \widetilde{m}^{\prime}\right)=0$.

Теперь перейдем к оценке второго члена в формуле (9.10). Он содержит множитель $\left|f^{(\tau)}(z, x)\right| / \tau$ ! для нашей функции $f(z, \zeta)$. Для ее производных мы имеем формулу

$$
\begin{aligned}
& \frac{f_{j}^{(\tau)}(z, x)}{\tau !}=\sum_{\left|\tilde{\tau}^{\prime}\right|=\tau} \sum^{(s)} \sum_{l_{0}=0}^{L_{0}} p_{l_{0}, \bar{l}(\bar{\lambda})} \frac{\Delta^{\left(\tau_{0}\right)}\left(N z, l_{0}, H_{0}, m_{0}\right)}{\tau_{0} !} \\
& \quad \times \Delta(\tilde{\nu}(\bar{l}), \tilde{m})\left(\frac{\tilde{\chi}(\bar{l})}{2^{s} b_{n}}\right)^{\tilde{\tau}}(\ln \tilde{\alpha})^{\tilde{\tau}} \tilde{\alpha}^{\tilde{\chi}(\bar{l}) x /\left(2^{s} b_{n}\right)}(\tilde{\tau} !)^{-\tilde{1}}\left(\prod_{\sigma \in \mathscr{S}}\left|\Phi_{s}\left(x, \widetilde{m}^{\prime}\right)\right|_{\sigma}\right)^{1 / \varkappa} .
\end{aligned}
$$

Если заменить здесь $\tilde{\alpha} \tilde{\chi}(\bar{l}) x /\left(2^{s} b_{n}\right)$ на $\bar{\alpha}^{\bar{l} x / 2^{s}}$, то при условии, что $\tau+\left|\widetilde{m}^{\prime}\right| \leqslant$ $M(x)$, все слагаемые для каждого $\tilde{\tau}^{\prime}$ будут равны нулю, а поправочньй член оценивается с помощью (10.3).

Показательная часть выражений, а также знаменатель оцениваются, как и ранее. При оценке полиномиальной части мы будем использовать лемму 7.3. Оценка (7.8) для многочленов и их производных одна и та же (при надлежашем выборе $\varepsilon_{3}$ в (8.16)). Также отметим, что при $|z| \leqslant Z_{s}$ мы имеем $|z| / 2^{s} \leqslant Z_{0}$ и в формуле (8.14) это принято во внимание. Таким образом, полиномиальная часть $f_{j}^{(\tau)}(z, x) / \tau$ ! оценивается, как в $\S 8$.

В дифференциальной части появились новые множители. Оценим их более аккуратно. Для $\Delta(\tilde{\nu}(\bar{l}), \tilde{m})$ имеем

$$
|\Delta(\tilde{\nu}(\bar{l}), \widetilde{m})| \leqslant \frac{\left(|\widetilde{m}|+\left(B_{1}+B_{0}\right) M_{0}\right)^{|\widetilde{m}|}}{|\widetilde{m}| !} .
$$

Аналогично, используя (3.1), получаем

$$
\sum_{|\tilde{\tau}|=\mu}\left|\left(\frac{\tilde{\chi}(\bar{l})}{b_{n}}\right)^{\tilde{\tau}} \frac{(\ln \tilde{\alpha})^{\tilde{\tau}}}{(\tilde{\tau} !)^{\tilde{1}}}\right| \leqslant \frac{\left(\left(B_{2}+B_{3}\right) M_{0}\right)^{\mu}}{\mu !},
$$

где $B_{2}, B_{3}$ определяются по формулам, аналогичным (5.13):

$$
\begin{aligned}
B_{3} & =\frac{\max \left\{\sum_{j=1}^{n-1}\left|\ln \left(\alpha_{j}\right) \frac{w_{j} b_{n}-w_{n} b_{j}}{b_{n}}\right|: \bar{w} \in \mathscr{W}\right\}}{M_{0}}, \\
B_{2} & =\frac{\max \left\{\sum_{j=1}^{n-1}\left|\ln \left(\alpha_{j}\right) \frac{w_{j} b_{n}-w_{n} b_{j}}{b_{n}}\right|: \bar{w} \in[-0.5,0.5]^{n}\right\}}{M_{0}} .
\end{aligned}
$$


Теперь введем для $W$ более сильное условие, чем (8.10):

$$
W \geqslant \ln \left(e\left(1+B_{0}+B_{1}+B_{2}+B_{3}\right)\right)
$$

Это дает нам оценку, аналогичную оценке (8.15):

$$
\begin{aligned}
& \sum_{|\tilde{\tau}|=\mu}\left|\Delta(\tilde{\nu}(\bar{l}), \tilde{m})\left(\frac{\tilde{\chi}(\bar{l})}{b_{n}}\right)^{\tilde{\tau}} \frac{(\ln \tilde{\alpha})^{\tilde{\tau}}}{(\tilde{\tau} !)^{\tilde{1}}}\right| \leqslant\left(|\widetilde{m}|+\left(B_{1}+B_{0}\right) M_{0}\right)^{|\widetilde{m}|} \frac{\left(\left(B_{2}+B_{3}\right) M_{0}\right)^{\mu}}{|\widetilde{m}| ! \mu !} \\
& \leqslant \frac{\left(M_{0}\left(1+B_{0}+B_{1}+B_{2}+B_{3}\right)\right)^{|\widetilde{m}|+\mu}}{(|\tilde{m}|+\mu) !} \leqslant \frac{\exp \left(c_{0} L_{0}\right)}{\left(2 \pi M_{0}\right)^{1 / 2}} .
\end{aligned}
$$

Таким образом, изменив условие для $W$, мы получили для $\left|f_{j}^{(\tau)}(z, x)\right| / \tau$ !, $x \in \mathscr{X}_{s}, \tau \leqslant M(x)$, ту же самую оценку (9.7), что и для самой функции $|f(z, x)|$, но с дополнительным множителем $1.1 \cdot\left|\Lambda l_{n} \zeta /\left(2^{s} b_{n}\right)\right|$ из (10.3), если принять индуктивную гипотезу, что для ранее рассмотренных точек выполняется (10.1) или (10.2):

$$
\left|\frac{f_{j}^{(\tau)}(z, x)}{\tau !}\right| \leqslant 0.22\left|\frac{\Lambda l_{n} x}{2^{s} b_{n}}\right| \exp \left(a_{1 s j}+a_{2 s j}|z|+a_{4}|x|\right)
$$

(слагаемое $a_{3} \ln \left(R / z_{0}\right)$ в показателе нужно только для больших $\left.\zeta \in \Gamma(0, R)\right)$. Поскольку в (9.10) мы имеем $r \leqslant 1$, то мы можем сделать второе слагаемое малым, если примем гипотезу о малости $|\Lambda|$ :

$$
1>\left|\frac{\Lambda Z_{0} U l_{n}}{b_{n}}\right| \exp \left(a_{1 s 2}+a_{2 s 2} 2 X_{s}+a_{4 s} Z_{s}\right)\left|\frac{Q\left(z_{0}\right)}{Q(\zeta)}\right|
$$

для всех встречающихся $U, z_{0}, l_{n}, \zeta$. Это завершает доказательство шага индукции.

Остаток параграфа будет посвящен выводу верхних оценок для $U=\operatorname{deg} Q(\zeta)$ и $V=\ln \left|Q\left(z_{0}\right) / Q(\zeta)\right|$ во втором слагаемом интерполящионной формулы. Поскольку, проводя индукцию, мы добавляли новые точки и увеличивали кратности, то достаточно рассмотреть только вторую часть $(j=2)$, где кратности задаются формулой $(10.10)$, и взять $\delta_{0}=1, z_{0}=2^{n}\left(2 X_{s}+1\right)$.

С учетом обозначений $\S 9$ мы видим из (9.5), что

$$
U=\sum_{\tau=1}^{T} u_{\tau}, \quad V \leqslant \sum_{\tau=1}^{T} u_{\tau} \ln \left(\frac{2 e z_{0}}{\delta_{0} u_{\tau}}\right)
$$


Оценим $u_{\tau}=\operatorname{deg} q_{\tau}$. Положим

$$
\begin{gathered}
t_{1}=\eta_{0 s}+2, \quad t_{2}=t_{1}+\xi_{s} n \ln 2, \quad t_{3}=t_{2}+\eta_{s}, \\
\varphi(\tau)= \begin{cases}2^{n+1}\left(2 X_{s}+1\right), & 0 \leqslant \tau \leqslant t_{1} \\
2^{n+1}\left(2 X_{s}+1\right) \exp \left(\frac{\eta_{0 s}+2-\tau}{\xi_{s}}\right), & t_{1}<\tau \leqslant t_{2}, \\
2 X_{s}+1, & t_{2}<\tau \leqslant t_{3},\end{cases}
\end{gathered}
$$

и проверим, что

$$
T \leqslant t_{3}, \quad u_{\tau} \leqslant \varphi(\tau), \quad 1 \leqslant \tau \leqslant T .
$$

Оценка $T$ следует непосредственно из (10.10), так как здесь

$$
T=M_{s}-M_{s+1}+1
$$

Для оценки $u_{\tau}$ примем во внимание, что $q_{\tau}(\zeta)$ имеет нули $x \in \mathbb{Z}$ с кратностями в $Q(\zeta)$ не меньше $\tau$. Случай $\tau>t_{2}$ возможен, только если $M(x)$ определена с помощью (10.7), значит, $x \in \mathscr{X}_{s}$ и для этих $\tau$ мы имеем (10.14).

Пусть теперь $\tau \leqslant t_{2}$. Здесь $M(x)$ определена с помощью $(10.8)$ и $u_{\tau}=2 x+1$, где $x$ - наибольшее число, для которого

$$
M(x)-M_{s+1}+1 \geqslant \tau .
$$

Решая это неравенство, получаем

$$
x \leqslant 2^{n+1} X_{s} \exp \left(\frac{\eta_{0 s}+2-\tau}{\xi_{s}}\right),
$$

что дает нам (10.14) для $t_{1}<\tau \leqslant t_{2}$. Оценка для $\tau \leqslant t_{1}$ очевидна, так как всегда $x \leqslant 2^{n+1} X_{s}$.

С учетом (10.13), (10.14) мы можем написать

$$
U \leqslant \int_{0}^{t_{3}} \varphi(\tau) d \tau, \quad V \leqslant \int_{0}^{t_{3}} \varphi(\tau) \ln \left(\frac{2 e z_{0}}{\varphi(\tau)}\right) d \tau
$$

и простое интегрирование дает, что

$$
U \leqslant 2^{n+1}\left(2 X_{s}+1\right)\left(2+U_{0}\right), \quad U_{0}=\eta_{0 s}+\xi_{s}\left(1-2^{-n}\right)+\eta_{s} 2^{-n-1}
$$

и

$$
\begin{gathered}
V \leqslant 2^{n+1}\left(2 X_{s}+1\right)\left(2+V_{0}\right), \\
V_{0}=\eta_{0 s}+\xi_{s}\left(2-(2+n \ln 2) 2^{-n}\right)+\eta_{s}(1+(n+1) \ln 2) 2^{-n-1} .
\end{gathered}
$$

Кроме того, напомним, что мы можем оценивать $U, V$ только при $s \leqslant S_{1}$ с $S_{1}$ из (4.7), так как при $s>S_{1}$ числа $X_{s}, M_{s}$ не меняются и шаг индукции проходит автоматически. Единственное место, где встречается растущее по $s$ выражение это знаменатель в $z / 2^{s}$ у многочленов. Но его мы учли с самого начала, положив $N=2^{S}$ в (7.5), (8.1) и (8.14). 


\section{§ 11. Выбор основных параметров}

Здесь мы выберем значения введенных ранее параметров (кроме $\varepsilon$ ), чтобы обеспечить все шаги доказательства и согласовать их с параметрами из $\S 2$. Покажем сначала, как обеспечить заключительную часть доказательства (см. §4). Примем некоторые естественные предположения. Одно из них

$$
W \geqslant W_{0} \geqslant 2 c_{3}, \quad c_{0} \geqslant C_{0} \geqslant 2 c_{3} .
$$

Пусть также с некоторым $\varepsilon_{6}$ вьполняется

$$
\varepsilon_{6} \geqslant \frac{1}{L_{0}}, \quad \varepsilon_{6} \geqslant \frac{1}{M_{0}}
$$

Кратности $M_{s+1}$ из (4.6) должны быть согласованы с $M_{s+1}$ из (10.10). Это означает, что при $s \leqslant S_{1}$ должно быть

$$
\eta_{s}+\xi_{s} n \ln 2+\eta_{0 s} \leqslant \frac{M_{0}}{2^{s+1}\left(1+\varepsilon_{0}\right)}
$$

Но в соответствии с $(10.5),(10.6),(8.21)$ выполняется

$$
\begin{aligned}
2^{s+1} & \left(\eta_{s}+\xi_{s} n \ln 2+\eta_{0 s}\right) \\
& =2^{s+1}\left(\frac{a_{2 s 1}}{c_{3}}+\frac{a_{3}}{2 X_{s}}+\frac{3 a_{1 s 1}}{2 c_{3} X_{s}}+\frac{1}{X_{s}}+\frac{a_{2 s 1} n \ln 2}{2 c_{3}}+\frac{a_{1 s 1}}{4 c_{3} X_{s}}\right) \\
& =\frac{(2+n \ln 2) \vartheta L n D}{c_{3}}+\frac{L_{0}}{X_{0}}+\frac{2}{X_{0}}+\frac{3 c_{0} L_{0} D\left(3+\varepsilon_{2}+\varepsilon_{3}+\varepsilon_{4}\right)}{2 c_{3} X_{0}} \\
& <\frac{M_{0}(2+n \ln 2)}{c_{1} c_{3}}+\frac{M_{0}\left(10+3\left(\varepsilon_{2}+\varepsilon_{3}+\varepsilon_{4}\right)+2 \varepsilon_{6}\right)}{2 c_{2} c_{3}} .
\end{aligned}
$$

Теперь положим

$$
\begin{array}{ll}
c_{4}=(2+n \ln 2) \frac{n+1}{n}, & c_{1}=\left(1+\varepsilon_{0}\right) \frac{c_{4}}{c_{3}} \\
c_{5}=(n+1) \frac{10+3\left(\varepsilon_{2}+\varepsilon_{3}+\varepsilon_{4}\right)+2 \varepsilon_{6}}{2}, & c_{2}=\left(1+\varepsilon_{0}\right) \frac{c_{5}}{c_{3}} .
\end{array}
$$

Это дает соответствие между (10.10) и (4.6). Если $s>S_{1}$, то в обоих случаях также имеем $M_{s+1}=M_{s}$. Оценивая уменьшение кратностей, мы легко получаем

$$
M_{S} \geqslant M_{0}-\frac{M_{0}\left(1-2^{-S}\right)}{1+\varepsilon_{0}}=\frac{M_{0}\left(\varepsilon_{0}+2^{-S}\right)}{1+\varepsilon_{0}} .
$$


С учетом (11.1) такой выбор $c_{2}$ дает, что

$$
\frac{L_{0}}{2 X_{0}}<\frac{M_{0}}{n\left(1+\varepsilon_{0}\right)}
$$

Значит, справа в (4.12) мы имеем

$$
\frac{L_{0}}{\left|\mathscr{X}_{S}\right|}<\frac{M_{S}}{n}
$$

Для оценки оставшейся части справа в (4.11) используем параметр $E_{1}$ из (2.2), что дает нам

$$
L_{S, 1}+\cdots+L_{S, n-1} \leqslant L D \vartheta(n-1) \frac{E_{1}}{2^{S}} .
$$

Так что для проверки (4.11) достаточно иметь

$$
M_{0} \frac{\varepsilon_{0}+\frac{2^{-S}(n-1)}{n}}{1+\varepsilon_{0}} \geqslant L D \vartheta(n-1) \frac{E_{1}}{2^{S}}
$$

или, эквивалентно,

$$
\left(\frac{2^{S} \varepsilon_{0} n}{n-1}+1\right) \frac{c_{4}}{c_{3}} \geqslant E_{1}
$$

В частности, отсюда следует, что при $E_{1} \leqslant c_{4} / c_{3}$ можно взять $\varepsilon_{0}=0$. В предшествуюших работах так и делалось, а условие типа $E_{1} \leqslant c_{4} / c_{3}$ ограничивало снизу значения параметров $A_{j}$. Однако (4.11) можно выполнить за счет выбора $S$ и $\varepsilon_{0}$. В данной работе мы просто возьмем во всех случаях $\varepsilon_{0}=\varepsilon_{6}$ и $S$ удовлетворяющим условию

$$
2^{S} \geqslant E_{1}\left(1+\varepsilon_{0}\right) \frac{n-1}{n c_{1} \varepsilon_{0}} .
$$

Следуюшее условие, которое надо обеспечить, - это неравенство (9.8), которого мы не касались в $\S 10$. Теперь покажем, как его выполнить. С помошью (10.5) перепишем его в форме

$$
e c_{3} \exp \left(c_{3}\right) \leqslant \frac{a_{1}+a_{2} z_{1}}{a_{4} z_{0}}=\frac{a_{1 s j}+a_{2 s j} z_{1}}{a_{4 s} z_{0}} .
$$

Покажем сначала, что наименьшее значение справа достигается при $j=1$ (часть 1 шага индукции) и

$$
z_{1}=z_{0}=Z_{s}=2^{n+1} X_{s} .
$$

При $j=1$ худшее значение для $z_{1}$ очевидно. Осталось сравнить случай $j=1$ со случаем $j=2$, где

$$
z_{1}=2 X_{s}, \quad z_{0}=2^{n+1} X_{s}+0.5 \leqslant 2^{n+1} X_{s}\left(1+\frac{2^{-n-2}}{X_{0}}\right) .
$$


Нам надо показать, что

$$
\frac{a_{1 s 1}+a_{2 s 1} 2^{n+1} X_{s}}{a_{4 s} 2^{n+1} X_{s}} \leqslant \frac{a_{1 s 2}+a_{2 s 2} 2 X_{s}}{a_{4 s} 2^{n+1} X_{s}\left(1+\frac{2^{-n-2}}{X_{0}}\right)} .
$$

С учетом $(8.21),(10.5)$ и того, что $\left[\mathbb{L}_{1}: \mathbb{Q}\right] / \varkappa=D,\left[\mathbb{L}_{2}: \mathbb{Q}\right] / \varkappa=2^{n} D$, это неравенство можно переписать в виде

$$
\frac{1}{2^{n+2} c_{4}} \leqslant\left(3+\varepsilon_{2}+\varepsilon_{3}+\varepsilon_{4}\right)\left(2^{n}-1-2^{-n-2}\right),
$$

и оно становится очевидным ввиду (11.1), (11.3). Так что нам надо проверить (11.5) для $j=1$ и $z_{0}, z_{1}$ из (11.6).

Умножая (11.5) на $\vartheta^{*}=\varepsilon^{*}+\vartheta_{0}^{*}($ см. (5.11), (5.12)) и используя обозначения (10.5), можно переписать (11.5) в виде

$$
\left(\varepsilon^{*}+\vartheta_{0}^{*}\right) e c_{3} \exp \left(c_{3}\right) \leqslant \frac{c_{0} L_{0}\left(3+\varepsilon_{2}+\varepsilon_{3}+\varepsilon_{4}\right) D}{2^{n+1} L X_{0}}+\vartheta n D .
$$

Чтобы это вьполнить, потребуем

$$
\vartheta_{0}^{*} e c_{3} \exp \left(c_{3}\right)=\vartheta n D
$$

и

$$
\varepsilon^{*} e c_{3} \exp \left(c_{3}\right) \leqslant c_{0} L_{0}\left(3+\varepsilon_{2}+\varepsilon_{3}+\varepsilon_{4}\right) \frac{D}{2^{n+1} L X_{0}} .
$$

Ввиду обозначений (5.11), (8.21) последнее неравенство можно переписать в виде

$$
\begin{gathered}
E_{2} \text { Dnec }_{3} \exp \left(c_{3}\right) \leqslant \frac{M_{0}\left(3+\varepsilon_{2}+\varepsilon_{3}+\varepsilon_{4}\right)}{2^{n} c_{2}}, \\
E_{2}=\frac{\max \left\{\left| \pm \ln \left(\alpha_{1}\right) \pm \cdots \pm \ln \left(\alpha_{n}\right)\right|\right\}}{n D}
\end{gathered}
$$

и оно будет выполнено за счет того, что $M_{0}$ будет большим.

Мы рассматриваем (11.7) как условие, определяющее число $\vartheta_{0}^{*}$. Отметим, что здесь надо быть осторожным, так как условие (5.7), содержащее $\vartheta_{0}^{*}$, играет важную роль в задании множества $\mathscr{W}$ и поэтому влияет на $\vartheta$ из (5.8). Если мы хотим упростить нашу задачу, то надо поступить следующим образом. Возьмем тело $\mathscr{W}_{0}$, задаваемое теми же формулами, что и $\mathscr{W}$, но без условия (5.7). Оно не зависит от $\vartheta_{0}^{*}$. Для него с некоторым $\vartheta$ имеет место равенство $h\left(\bar{\alpha}, \mathscr{W}_{0}\right)=\vartheta$. Поскольку $\mathscr{W} \subseteq \mathscr{W}_{0}$, то по лемме 3.2 для $\mathscr{W}$ можно взять то же самое $\vartheta$. 
Начиная с этого момента, мы считаем, что множества $\mathscr{W}$ и $\mathscr{W}_{0}$ определены с помощью (5.5), (5.6), а $\mathscr{W}$ дополнительно с помощью (5.7). В соответствии с формулой произведения это дает нам, что мы имеем (5.9) с $\vartheta$ из (2.1). Отметим, что числа $B_{0}, B_{1}, B_{2}, B_{3}$ определены с помощью (5.13), (10.11) несколько точнее, чем в (2.7). Теперь применим лемму 5.1 с $\vartheta_{1}^{*}=E L n D / 2$ и $E$ из (2.2). Если мы возьмем

$$
\eta^{-1}=\frac{\vartheta_{1}^{*}}{L \vartheta_{0}^{*}}=e E c_{3} \frac{\exp \left(c_{3}\right)}{2 \vartheta} \geqslant 1
$$

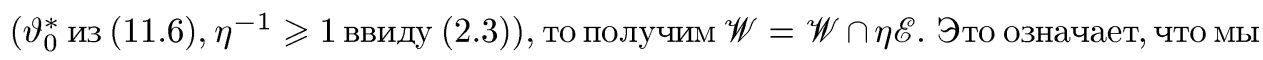
можем взять

$$
\Omega_{0}=\Omega\left(\operatorname{Eec}_{3} \frac{\exp \left(c_{3}\right)}{2 \vartheta}\right)^{\rho} \geqslant \Omega e^{n}
$$

При оценке линейных форм от логарифмов важно минимизировать $\omega_{0}$ и другие параметры из (8.21). Если $E$ мало, то выбор $c_{3}=C_{3}^{*}$ достаточно хорош. Если у нас нет информации о числах $\alpha_{j}$, кроме $A_{j} \geqslant \max \left\{h\left(\alpha_{j}\right),\left|\ln \alpha_{j}\right| / D\right\}$, то $\vartheta \leqslant 1$, $E \leqslant 1$, и мы можем взять $c_{3}=1$ или равной какой-либо другой константе. Именно так (правда, не столь явно) было в работах других авторов.

Основная идея данной работы, позволяющая устранить множитель $n^{n}$ из оценок, заключается в лучшем выборе $c_{3}$. При $c_{3}=n / \rho$ зависимость $\omega_{0}$ от $c_{3}$ будет иметь вид

$$
\frac{\left(c_{3} \exp \left(c_{3}\right)\right)^{\rho}}{c_{3}^{n}}=\left(\frac{n}{\rho}\right)^{\rho-n} e^{n}
$$

что дает $n^{n}$ в знаменателе и удаляет этот множитель из оценок.

Если мы хотим учесть возможную малость параметра $E$, то надо положить $c_{3}=$ $C_{3}$ с $C_{3}$ из (2.3). Тогда мы имеем при надлежашем выборе $\varepsilon_{0}$, что $c_{1}=C_{1}$ для $c_{1}$ из (11.3) и $C_{1}$ из (2.4). Возможны небольшие уточнения в выборе $c_{3}$, но в данной работе мы этого делать не будем.

Теперь покажем, как упростить условие (11.8).

Между параметрами $E_{2}$ и $E$ есть некоторая связь. Мы имеем

$$
E_{2} \leqslant \max \left\{\frac{\left|\ln \alpha_{j}\right|}{D}: 1 \leqslant j \leqslant n\right\} \leqslant n E A, \quad A=\max \left\{A_{j}: 1 \leqslant j \leqslant n\right\},
$$

так что для выполнения (11.9) достаточно иметь

$$
E A D n^{2} e c_{3} \exp \left(c_{3}\right) \leqslant \frac{3 M_{0}}{2^{n} c_{2}} .
$$

Поскольку ввиду (11.10), (8.21) $M_{0}$ содержит $\rho$ множителей, не меньших, чем $E e c_{3} \exp \left(c_{3}\right) /(2 \vartheta) \geqslant e^{n / \rho}$, мы можем упростить последнее неравенство, если уберем все $\varepsilon$ и используем (11.3). После таких изменений получается неравенство

$$
D\left(c_{1} D \vartheta\right)^{n-1}\left(\frac{\Omega}{A}\right)\left(\frac{c_{0}}{2 c_{3}}\right)\left(E e c_{3} \frac{\exp \left(c_{3}\right)}{2 \vartheta}\right)^{\rho-1} 6(6(2+n \ln 2)+5 n) \frac{(n+1) n^{n}}{2^{n} n^{3} n !} \geqslant 1 .
$$


Ввиду (11.1) оно следует из (2.11), а в случае теоремы 2.3 оно будет проверено прямым вычислением.

\section{§12. Доказательство теоремы 2.1}

Нам осталось выбрать малые $\varepsilon$, удовлетворяюшие соответствуюшим условиям, обеспечить $X_{0}, L_{0}, M_{0} \in \mathbb{Z}$ (см. (8.21)), выбрать $S$ из $\S 4, H_{0}$ из $\S 8$ и посмотреть, насколько мало должно быть $|\Lambda|$ для выполнения (10.4), (10.12). Положим для краткости

$$
\omega_{j}=\frac{\omega_{0}}{A_{j} c_{1} D \vartheta}, \quad 1 \leqslant j \leqslant n,
$$

с $\omega_{0}$ из (8.21). Отметим, что $\omega_{0}=\omega$, где $\omega-$ из (2.5). Ввиду (11.1), (2.9), (2.10), (11.1), (11.10) мы имеем

$$
D \omega_{j} \frac{\min \left\{c_{0}, W\right\}}{2 c_{3}} \geqslant 1, \quad 0 \leqslant j \leqslant n
$$

Положим (см. (8.8), (8.12), (8.16), (8.20), (8.21), (11.2))

$$
\begin{gathered}
\varepsilon_{0}=\varepsilon_{6}=\frac{e^{-2 n}}{148}, \\
1+\varepsilon_{1}=\left(1+\frac{(n+1) \varepsilon_{6}}{2}\right)^{n}, \\
\varepsilon_{2}=\frac{e^{-n}}{72}, \quad \varepsilon_{3}=\frac{e^{-n}}{91}, \\
\varepsilon_{4}=\frac{e^{-2 n}}{109}, \quad \varepsilon_{5}=\frac{1}{10 n+11} .
\end{gathered}
$$

Кроме того, введем вспомогательные функции

$$
\begin{gathered}
\xi_{1}=\xi_{1}(n)=\left(1+\varepsilon_{0}\right)\left(1+\varepsilon_{1}\right)\left(\left(2+\varepsilon_{5}\right)\left(3+\varepsilon_{2}+\varepsilon_{3}\right)+\left(1+\varepsilon_{5}\right) \frac{c_{5}}{c_{4}}\right) 4 c_{5} e^{n} \frac{n^{n}}{n !} \\
\xi_{0}=\xi_{0}(n)=\ln \xi_{1}(n), \quad \xi_{2}=\xi_{2}(n)=\left(\xi_{1}(n) \xi_{0}(n)\right)^{1 / 2}
\end{gathered}
$$

$\left(c_{4}, c_{5}\right.$ - из (11.3), так что $\xi_{0}, \xi_{1}, \xi_{2}$ не зависят от $\left.c_{3}\right)$. Легко видеть, что $\xi_{1}(n) / e^{2 n} \gg$ $n^{1 / 2}$, и простое вычисление для небольших $n$ доказывает, что наименьшее значение $\xi_{1}(n) / e^{2 n}(>148)$ достигается при $n=2$. Для больших $n$ эта же оценка получается за счет роста функции. Это дает

$$
\xi_{1}(n) \geqslant 148 e^{2 n}
$$


Кроме того, легко проверить, что для $C_{2}$ из (2.4) имеем

$$
1 \leqslant \frac{C_{2}}{\frac{\xi_{1}(n)}{2 c_{3}}} \leqslant 1.03
$$

Иначе говоря, $C_{2}$ - это упрошенное выражение для $\xi_{1} /\left(2 c_{3}\right)$.

Теперь оценим $X_{0}$ из (8.21) с $c_{2}$ из (11.3). Ввиду (11.1) мы имеем $X_{0}>10(n+1)$, значит, $X_{0} \geqslant 10 n+11$ и $\varepsilon_{5}$ удовлетворяет (8.21). Чтобы получить $X_{0} \in \mathbb{Z}$, отметим, что $W_{0}$ из (2.7) удовлетворяет тем же условиям, что и $W$, и затем возьмем $W=W_{0}\left(1+\varepsilon_{X}\right)$ с некоторым $\varepsilon_{X}$, удовлетворяющим условию

$$
0 \leqslant \varepsilon_{X} \leqslant \varepsilon_{7}=\frac{1}{2 c_{5}}
$$

Это позволяет обеспечить $X_{0} \in \mathbb{Z}$. Множитель $\left(1+\varepsilon_{7}\right)$ будет добавлен к конечной оценке $|\Lambda|$. Легко видеть, что мы можем взять

$$
\begin{gathered}
L_{0}=\left[\frac{D \omega_{0} \xi_{1} W}{2 c_{3}}\right]+1=\left(1+\varepsilon_{L}\right) \frac{D \omega_{0} \xi_{1} W}{2 c_{3}}, \\
M_{0}=\left(1+\varepsilon_{L}\right) \frac{D \omega_{0} \xi_{1} c_{0}}{2 c_{3}} \\
\frac{L}{A_{j}}=\left(1+\varepsilon_{L}\right) D \omega_{j}\left(\frac{\xi_{1}}{n}\right) \frac{c_{0}}{2 c_{3}},
\end{gathered}
$$

чтобы получить $L_{0} \in \mathbb{Z}, M_{0} \in \mathbb{Z}$, где $\varepsilon_{L}$ и $c_{0}$ удовлетворяют

$$
0 \leqslant \varepsilon_{L} \leqslant \varepsilon_{6}, \quad C_{0} \leqslant c_{0} \leqslant\left(1+\varepsilon_{6}\right) C_{0}
$$

$C_{0}$ - из $\S 2$, при этом (2.6) дает, что $C_{0}$ удовлетворяет всем условиям для $c_{0}$. Ввиду $(12.1),(12.3),(12.4)$ мы также имеем (11.2). Множитель $\left(1+\varepsilon_{6}\right)^{2}$ следует добавить к окончательной оценке $|\Lambda|$. После этого определим числа

$$
\begin{gathered}
L_{n}^{\prime}=\left(1+\varepsilon_{L}\right) \xi_{1} \max \left\{\frac{D \omega_{n} c_{0}}{2 c_{3}}, \frac{c_{0}}{2 c_{3}}, \frac{E_{1} c_{3}}{c_{4}}\right\} \\
S=\left[\log _{2}\left(\frac{2 L_{n}^{\prime}}{n}\right)\right], \quad H_{0}=\left[\frac{\xi_{2} W}{C_{0}}\right]+1, \quad L_{-1}=\left[\frac{L_{0}}{H_{0}}\right] .
\end{gathered}
$$

Отметим, что $S$ удовлетворяет (11.4) и $S \geqslant\left[\log _{2}\left(2 L_{0, n}\right)\right]$.

Для $c_{0}$ нам нужно неравенство

$$
c_{0} \geqslant \ln \left(\left(1+\varepsilon_{6}\right) D L_{n}^{\prime}\right) \geqslant \ln \left(\left(1+\varepsilon_{6}\right) \xi_{1} D\right) \geqslant \xi_{0},
$$


которое следует из (2.5), (12.5), (12.8).

Теперь можно проверить условия (8.13), (8.14). Используя вышеприведенные неравенства, мы получаем в (8.13), что

$$
\begin{aligned}
& \ln \left(2.8361 H_{0} \frac{M_{0}+L_{-1}+1}{L_{0}}\right) \\
& \quad \leqslant \ln \left(2.8361\left(\xi_{2}+1\right)\left(1+\frac{1}{\xi_{1}}+\frac{1}{\xi_{2}}\right) \frac{c_{0}}{2 c_{3}}\right) \leqslant \ln \left(L_{n}^{\prime}\right) \leqslant c_{0} .
\end{aligned}
$$

Доказывая (8.14), мы аналогично получаем

$$
\begin{aligned}
& \ln \left(e\left(1+\frac{N\left(Z_{0}+0.5\right)}{H_{0}}\right)\right) \\
& \quad \leqslant \ln \left(D L_{n}^{\prime}\left(1+\varepsilon_{6}\right)\left(1+\varepsilon_{5}\right) e 2^{n+2} \frac{2 c_{5}}{\xi_{2}}\right) \leqslant \ln \left(D L_{n}^{\prime}\left(1+\varepsilon_{6}\right)\right) \leqslant c_{0}
\end{aligned}
$$

$\left(N, Z_{0}-\right.$ из $\left.(8.5),(10.8)\right)$. Здесь мы пользовались неравенствами

$$
\begin{aligned}
& \xi_{1} \geqslant 2.8361\left(\xi_{2}+1\right)\left(1+\frac{1}{\xi_{1}}+\frac{1}{\xi_{2}}\right), \\
& \xi_{2} \geqslant\left(1+\varepsilon_{5}\right) e 2^{n+2} \frac{2 c_{5}}{\xi_{2}}
\end{aligned}
$$

которые можно проверить численно.

Следующим шагом проверим, что числа $\varepsilon$ из (12.2) удовлетворяют неравенствам, в которых они были введены. На самом деле в (12.2) даны упрощенные выражения, более точные будут получены попутно. Неравенства для $\varepsilon_{6}(11.2)$ сразу следуют из (12.4) (нужно только, чтобы было $\varepsilon_{6} \geqslant 1 / \xi_{1}$ ). Далее проверим, что $\varepsilon_{2}$ удовлетворяет (8.12). Ввиду (7.2), (7.7), (12.10) достаточно проверить, что

$$
\varepsilon_{2} \geqslant \frac{\ln \left(2 \pi H_{0}\right)}{2 c_{0} H_{0}}+\frac{1}{12 c_{0} H_{0}^{2}}
$$

Мы будем несколько раз использовать простые неравенства $x \leqslant e^{x / e}, \ln x \leqslant$ $x / e, x>0$. Ввиду (12.3), (12.9) легко получается, что

$$
\frac{1}{12 c_{0} H_{0}^{2}} \leqslant \frac{1}{12 \xi_{0} \xi_{2}^{2}}
$$

Чтобы завершить доказательство (8.12), положим

$$
x=\frac{\frac{\pi \xi_{2} W}{c_{3}}}{D L_{n}^{\prime}} \leqslant \frac{\frac{\pi \xi_{2} W}{c_{3}}}{\xi_{1}} .
$$


Тогда неравенства (11.1), (12.4), (12.10), (12.12) дают

$$
\frac{\ln \left(2 \pi H_{0}\right)}{2 c_{0} H_{0}} \leqslant \frac{\ln \left(\frac{2 \pi \xi_{2} W}{2 c_{3}}\right)}{\frac{2 c_{0} \xi_{2} W}{2 c_{3}}}=\frac{\ln \left(D L_{n}^{\prime}\right)+\ln x}{\frac{2 c_{0} \xi_{2} W}{2 c_{3}}} \leqslant \frac{1}{2 \xi_{2}}+\frac{x}{e c_{0} \xi_{2} \frac{W}{c_{3}}} \leqslant \frac{1}{2 \xi_{2}}+\frac{\pi}{e \xi_{0} \xi_{1}} .
$$

Так что для вьполнения (8.12) достаточно выполнения неравенства

$$
\varepsilon_{2} \geqslant \frac{1}{2 \xi_{2}}+\frac{\pi}{e \xi_{0} \xi_{1}}+\frac{1}{12 \xi_{0} \xi_{2}^{2}}
$$

которое легко следует из (12.13).

Теперь проверим, что $\varepsilon_{3}$ из (12.3) удовлетворяет условию (8.16). Сначала, используя $(5.10),(8.21)$ и обозначения из $(5.10),(8.21)$, оценим $\left|\mathscr{L}_{0}\right|$. Мы имеем

$$
\frac{L^{n}}{\Omega_{0}}=\frac{M_{0}^{n}}{\Omega_{0}\left(c_{1} n D \vartheta\right)^{n}}=\left(\frac{\left(1+\varepsilon_{L}\right) \xi_{1}}{e n}\right)^{n} \omega_{0}^{n-1} \frac{D c_{0}}{2 c_{3}} .
$$

Принимая во внимание малость $\varepsilon_{6}$, получаем

$$
\left|\mathscr{L}_{0}\right| \leqslant \frac{L_{n}}{\Omega_{0}}+1 \leqslant \frac{\left(1+\left(n e \varepsilon_{6}\right)^{n}\right) L^{n}}{\Omega_{0}}<\frac{\left(1+\varepsilon_{6}\right) L^{n}}{\Omega_{0}} .
$$

Значит, для $J=\left(L_{0}+1\right)\left|\mathscr{L}_{0}\right|$ из $(8.6)$ мы имеем

$$
\begin{aligned}
J & \leqslant\left(1+\varepsilon_{6}\right)^{2} D \omega_{0} \xi_{1}\left(\frac{L^{n}}{\Omega_{0}}\right) \frac{W}{2 c_{3}} \\
& \leqslant\left(\left(1+\varepsilon_{6}\right) \frac{\omega_{0} c_{0}}{2 c_{3} e n}\right)^{n}\left(\left(1+\varepsilon_{6}\right) \xi_{1} D\right)^{n+1} \frac{W}{2 c_{3}} \\
& \leqslant D^{-n}\left(\left(1+\varepsilon_{6}\right) \frac{D \omega_{0}}{2 c_{3} e n}\right)^{n}\left(\left(1+\varepsilon_{6}\right) \xi_{1} D\right)^{n+1} \frac{W}{2 c_{3}} \\
& \leqslant D^{-n} \exp \left(\frac{W}{2 c_{3} e}+\left(1+\varepsilon_{6}\right) \frac{D \omega_{0}}{e^{2} n}+(n+1) c_{0}\right) \\
& \leqslant D^{-n} \exp \left(c_{0} L_{0} \frac{\frac{1}{e}+\frac{1+\varepsilon_{6}}{e^{2} n}+\frac{n+1}{2 c_{3}}}{\xi_{1}}\right) .
\end{aligned}
$$

Сумма в (8.16) оценивается с учетом (7.9) как

$$
\sum_{l_{0}=0}^{L_{0}} \sum_{\tau+m_{0} \leqslant l_{0}} \delta\left(l_{0}, m_{0}, \tau\right) \frac{20}{\left(2 \pi M_{0}\right)^{1 / 2}} \leqslant e^{\left(H_{0}-1\right) / e} \leqslant e^{c_{0} L_{0} /\left(e \xi_{2}\right)} .
$$


Это вместе с (12.11) ввиду $c_{3} \geqslant n / \rho \geqslant n / 2$ дает нам, что для выполнения (8.16) достаточно иметь

$$
\varepsilon_{3} \geqslant \frac{1}{e \xi_{2}}+\frac{\frac{1}{e}+\frac{1+\varepsilon_{6}}{e^{2} n}+1+\frac{1}{n}}{2 \xi_{1}} .
$$

Но условие (12.3) для $\varepsilon_{3}$ сильнее.

Осталось проверить (8.20) для $\varepsilon_{4} \mathrm{c} \gamma$ из (6.4). Так как $J$ оценивается с помошью (12.11), надо оценить только $|\Delta(\mathbb{K})|^{1 / 2 D_{\mathbb{K}}}$. Поскольку (12.11) содержит справа $D^{-n} \leqslant 2 D_{\mathbb{K}}^{-1}$, то при использовании (6.6) надо только оценить $D_{\mathbb{K}} h\left(\alpha_{j}\right)$. Ввиду (2.1) мы имеем

$$
\begin{aligned}
D_{\mathbb{K}} h\left(\alpha_{j}\right) & \leqslant \varkappa D n \vartheta A_{j} \leqslant \varkappa n D \vartheta A_{j} D \omega_{j} \frac{W}{2 c_{3}}=\varkappa n D \omega_{0} \frac{W}{2 c_{3} c_{1}} \\
& \leqslant \varkappa n D \omega_{0}\left(\frac{c_{3}}{c_{4}}\right) \frac{W}{2 c_{3}} \leqslant c_{0} L_{0} \varkappa \frac{n}{2 c_{4} \xi_{1}},
\end{aligned}
$$

и вместе с (12.11) это дает, что для $\varepsilon_{4}$ достаточно иметь

$$
\varepsilon_{4} \geqslant \frac{n}{c_{4} \xi_{1}}+\frac{\frac{1}{e}+\frac{1+\varepsilon_{6}}{e^{2} n}+1+\frac{1}{n}}{2 \xi_{1}} .
$$

Условие (12.3) для $\varepsilon_{4}$ также сильнее.

В конще этого параграфа проверим неравенства из $\S 10$, где было использовано предположение о малости линейной формы от логарифмов. Это было дважды: при оценке первого слагаемого в интерполяционной формуле в (10.4) и второго слагаемого в (10.12).

Сначала посмотрим, насколько малым должно быть $|\Lambda|$, чтобы обеспечить (10.12). Оценим составляюшие в показателе этого условия, сравнивая их с выражением

$$
F=2^{n} D c_{0} L_{0}
$$

Используя (10.5), (10.6), (8.21), (11.3), мы имеем

$$
a_{1 s 2}=\left(3+\varepsilon_{4}+\varepsilon_{6}\right) F, \quad a_{2 s 2} 2 X_{s}=\left(\frac{2 c_{5}}{c_{4}}\right) F .
$$

Для оценки $a_{4 s} Z_{s}$ используем $(10.8),(5.12),(11.7),(11.8)$. Это дает

$$
\begin{aligned}
a_{4 s} Z_{s} & =2^{n+1} \vartheta^{*} L X_{0}=2^{n+1}\left(\vartheta_{0}^{*}+\varepsilon^{*}\right) L X_{0} \\
& \leqslant \frac{2^{n+1} \vartheta n D L X_{0}+c_{0} L_{0} D\left(3+\varepsilon_{2}+\varepsilon_{3}+\varepsilon_{4}\right)}{e c_{3} \exp \left(c_{3}\right)} \\
& \leqslant 2 \frac{\left(\frac{c_{5}}{c_{4}}+3+\varepsilon_{2}+\varepsilon_{3}+\varepsilon_{4}\right) F}{e c_{3} \exp \left(c_{3}\right)} .
\end{aligned}
$$


Для оценки $V=\ln \left|Q\left(z_{0}\right) / Q(\zeta)\right|$ используем (10.15). Напомним, что мы можем рассматривать только $s \leqslant S_{1}$ с $S_{1}$ из (4.7). Тогда

$$
\begin{aligned}
V_{s 1}= & 2^{n+3} X_{s} \leqslant 2^{n+3+s} X_{0} \leqslant 2^{n+3} X_{0} M_{0} \leqslant 4 c_{2} F, \\
V_{s 2}= & 2^{n+2} X_{s} \eta_{0 s}=\left(3+\varepsilon_{2}+\varepsilon_{3}+\varepsilon_{4}\right) \frac{F}{c_{3}} \\
V_{s 3}= & 2^{n+2} X_{s} \xi_{s}=2^{n+2} X_{0} \vartheta L n \frac{D}{2 c_{3}}=\frac{2 c_{5}}{c_{3} c_{4}} F, \\
V_{s 4}= & 2^{n+2} X_{s} \eta_{s}=2 V_{s 3}+2 V_{s 2}+2^{n+1} L_{0}+2^{n+2} \\
\leqslant & 2 V_{s 2}+2 V_{s 3}+F\left(\frac{2}{\ln \left(\xi_{1}\right)}+\frac{4}{\xi_{2}^{2}}\right) \\
V \leqslant & \left(1+\frac{\varepsilon_{5}}{2}\right)\left(V_{s 1}+V_{s 2}+V_{s 3}\left(2-(2+n \ln n) 2^{-n}\right)\right. \\
& \left.+V_{s 4}(1+(n+1) \ln 2) 2^{-n-1}\right) .
\end{aligned}
$$

Аналогично с помощью (10.15) получается оценка для $U$ :

$$
U \leqslant\left(1+\frac{\varepsilon_{5}}{2}\right)\left(V_{s 1}+V_{s 2}+V_{s 3}\left(1-2^{-n}\right)+V_{s 4} 2^{-n-1}\right)=c_{6} F
$$

с некоторым параметром $c_{6}=c_{6}(n)$, который можно получить из (12.15). Отметим, что $c_{6}$ содержит $c_{3}$ в знаменателе некоторых слагаемых. Так что мы можем упростить $c_{6}$, заменив $c_{3}$ на $n / 2 \leqslant n / \rho \leqslant c_{3}$. Легко видеть, что $c_{6} \ll 1$, а компьютерная проверка показьвает, что наибольшее значение $c_{6}(<71)$ достигается при $n=2$. Тогда из (12.16), (12.12), (12.7), (12.10), (12.5) мы имеем

$$
\begin{aligned}
\ln U & \leqslant n \ln 2+\ln \left(c_{6}\right)+\ln \left(D W \omega_{0}\right)+\ln \left(\left(1+\varepsilon_{6}\right) \frac{\xi_{1} c_{0}}{2 c_{3}}\right) \\
& \leqslant n \ln 2+\ln \left(c_{6}\right)+\frac{D W \omega_{0}}{e}+c_{0} \leqslant 2^{-n} \varepsilon_{6}\left(n \ln 2+\ln \left(c_{6}\right)+\frac{1}{e}+1\right) F .
\end{aligned}
$$

Еще одно выражение в (10.12) - это $\left|Z_{0} l_{n} / b_{n}\right|$, которое ввиду (8.14), (12.5), (12.9) может быть оценено как

$$
\begin{aligned}
\ln \left|\frac{Z_{0} l_{n}}{b_{n}}\right| & \leqslant \ln \left(\frac{e Z_{0} N}{H_{0}}\right)+\ln \left(\left(\xi_{2}+1\right) \frac{W}{2 c_{3} e}\right) \\
& \leqslant c_{0}+\frac{W}{2 c_{3} e^{2}}+\ln \left(\xi_{1}(n)\right) \frac{\xi_{2}+1}{e \xi_{1}} \leqslant F 2^{-n} \frac{2+e^{-2}+\xi_{2}}{\xi_{1}} .
\end{aligned}
$$

Заменяя выражения, входящие в (10.12), на их оценки (12.11)-(12.18), мы видим, что для выполнения (10.12) достаточно иметь

$$
\ln |\Lambda|<-c_{7} F
$$


с некоторым параметром $c_{7}$, который можно явно выписать.

Теперь перейдем к изучению условия (10.4). Оно намного проще, чем (10.12), но содержит $\left|\zeta / 2^{s}\right| \mathrm{c}|\zeta|=R=\left(U-a_{3}\right) / a_{4}$ из (9.7). Поэтому нам надо найти нижнюю оценку для $a_{4 s} 2^{s}$. Это может быть сделано аналогично выводу (12.14):

$$
a_{4 s} 2^{s}=\vartheta_{0}^{*} L \geqslant \frac{M_{0}}{c_{1} e c_{3} \exp \left(c_{3}\right)} \geqslant \exp \left(-c_{0}\right) \geqslant \exp \left(-\varepsilon_{6} F\right)
$$

Отсюда видно, что (10.4) - более слабое условие, чем (10.12).

Осталось вернуться к исходным обозначениям $C_{0}, W_{0}$ из $\S 2$. Из $(12.6)-(12.9)$ видно, что (12.19) следует из

$$
\ln |\Lambda|<-\left(1+\varepsilon_{7}\right)\left(1+\varepsilon_{6}\right)^{2} \xi_{1} c_{7} 2^{n} C_{0} \omega D \frac{W_{0}}{2 c_{3}}
$$

А это ведет к противоречию. Таким образом, (12.20) неверно.

Компютерная проверка показывает, что выполняется (12.5) и

$$
\left(1+\varepsilon_{7}\right)\left(1+\varepsilon_{6}\right)^{2} c_{7} \leqslant 91
$$

Так что с учетом (12.5) отрицание (12.20) влечет (2.12), а это доказывает теоремy 2.1.

\section{§13. Доказательство теоремы 2.2}

Из (2.13) следует, что $\vartheta=1, E=1, C_{3}=n / \rho$ удовлетворяют соответственно условиям (2.1), (2.2), (2.3). В (2.6) также имеем

$$
\frac{D \omega}{C_{1} \vartheta A_{n}} \geqslant 1 \geqslant \frac{E_{1}}{C_{1}}
$$

Положим для $C_{0}^{\prime}$ из $(2.15) C_{0}=1.23 C_{0}^{\prime}$ и покажем, что такое $C_{0}$ удовлетворяет (2.6). Из (2.4), (2.11) мы, очевидно, имеем

$$
C_{0} \geqslant C_{0}^{\prime} \geqslant \ln \left(\frac{C_{2} D \omega}{C_{1} A_{n}}\right) \geqslant 2 n \geqslant \frac{2 n}{\rho}=2 C_{3} .
$$

Так что остается проверить, что $0.23 C_{0}^{\prime} \geqslant \ln C_{0}=\ln \left(1.23 C_{0}^{\prime}\right)$. Написав $C_{2}=C_{2}(n)$ и использовав то, что $C_{0} \geqslant \ln C_{2}(n)$, имеем

$$
\frac{\ln \left(1.23 C_{0}^{\prime}\right)}{C_{0}^{\prime}} \leqslant \frac{\ln \left(1.23 \ln C_{2}(n)\right)}{\ln C_{2}(n)} \leqslant \frac{\ln \left(1.23 \ln C_{2}(2)\right)}{\ln C_{2}(2)} \leqslant 0.23
$$


что доказывает (2.6).

Для $C_{0}$ мы также имеем $(2.11),(2.9),(2.10)$.

Остается показать, что в (2.8) можно взять $W_{0}=\ln (2 e B)$ с $B$ из (2.14). Так как $b_{n} \neq 0$, то $B \geqslant 1$. Теперь оценим составляющие $W_{0}$ из $(2.7)$. Используя $(2.10),(2.13)$, получаем

$$
\frac{\left|b_{j}\right|}{\omega}=\frac{\left(b_{j} \mid A_{j} / A_{n}\right.}{A_{j} C_{1} D / \omega_{n}} \leqslant B, \quad 1 \leqslant j \leqslant n
$$

значит, $B_{0}<n B / C_{2}<B /(8 n)$. Затем мы имеем

$$
\frac{\left|b_{n}\right|}{A_{j} C_{1} D}=\frac{\left|b_{n}\right| A_{n} / A_{n}}{A_{j} C_{1} D} \leqslant B, \quad 1 \leqslant j \leqslant n-1,
$$

и также

$$
\frac{\left|b_{j}\right|}{A_{n} C_{1} D}=\frac{\left|b_{j}\right| A_{j} / A_{n}}{A_{j} C_{1} D} \leqslant B, \quad 1 \leqslant j \leqslant n-1,
$$

значит, $B_{1} \leqslant(n-1) B / n$. Мы также имеем

$$
\frac{\left|\ln \alpha_{j}\right|}{\omega}=\frac{\frac{\left|\ln \alpha_{j}\right|}{D A_{j}}}{\omega_{j} C_{1}} \leqslant \frac{1}{C_{1}} \leqslant \frac{B}{C_{1}}
$$

и

$$
\frac{\left|b_{j} \ln \alpha_{j}\right|}{\omega}=\left|\frac{b_{j} A_{j}}{A_{n}}\right| \frac{\frac{\left|\ln \alpha_{j}\right|}{D A_{j}}}{\omega_{j} C_{1}} \leqslant \frac{B}{C_{1}}
$$

значит, $B_{2} \leqslant n B /\left(C_{1} C_{2}\right) \leqslant B /(8 n)$. Наконец, мы имеем $\left|\ln \alpha_{j}\right| /\left(D A_{j}\right) \leqslant 1 \leqslant B$, и также

$$
\frac{\left|b_{j} \ln \alpha_{j}\right|}{D A_{n}}=\left(\frac{\left|b_{j}\right| A_{j}}{A_{n}}\right) \frac{\left|\ln \alpha_{j}\right|}{D A_{j}} \leqslant B,
$$

значит, $B_{3} \leqslant B(n-1) / n$.

Собирая вместе эти неравенства, мы видим, что можно взять $W_{0}=$ $\max \{2 n / \rho, \ln (e(1+2 B(1-1 /(8 n))))\}$. Это еще не совсем то, что нам надо, но если $B$ достаточно велико, то это дает теорему 2.2. Так что остается показать, что без ограничения общности мы можем допустить $\ln (2 e B) \geqslant 2 n$.

Параметр $B$ вида (2.14) очень удобен для написания оценок типа лиувиллевых.

ЛЕмма 13.1. Пусть $\Lambda-$ из (1.2), $A_{j} \geqslant h\left(\alpha_{j}\right), 1 \leqslant j \leqslant n, A_{n}>0$, $\Lambda /(2 \pi i) \notin \mathbb{Z}, B-$ из (2.14). Тогда

$$
\left|e^{\Lambda}-1\right| \geqslant 2^{1-D} \exp \left(-n D B A_{n}\right)
$$


ДоКАЗАТЕЛЬСТВо. Оценка немедленно получается из формулы произведения для алгебраического числа $\gamma=e^{\Lambda}-1=\bar{\alpha}^{\bar{b}}-1 \neq 0$.

Теперь допустим, что (2.12) не выполняется. Тогда $|\Lambda|<0.1$ и для $\gamma$ из леммы 13.1 мы имеем $|\gamma| \leqslant 1.1|\Lambda|$. Это дает

$$
\begin{aligned}
& -91 \cdot 2^{n} C_{2} C_{0} D^{n+2} \omega \ln (2 e B) \geqslant \ln |\Lambda| \\
& \quad \geqslant \ln |\gamma|-\ln (1.1) \geqslant(1-D) \ln 2-n D B A_{n}-\ln 1.1 .
\end{aligned}
$$

Поскольку $C_{2}$ из (2.4) велико, это дает

$$
n D B A_{n} \geqslant 90 \cdot 2^{n} C_{2} C_{0} D^{n+2} \omega W_{0},
$$

или

$$
B \geqslant 90 \cdot 2^{n} C_{2} C_{0} \frac{D^{n+1} \omega}{A_{n} n} W_{0} .
$$

В частности, $B \geqslant C_{2} D^{2} \omega_{n} C_{0} \geqslant e^{2 n}$ и для $W_{0}=\ln (2 e B)$ мы имеем $(2.8),(2.9)$, (2.10), что доказывает теорему 2.2 .

ЗАМЕЧАНИЕ. Из (13.1) видно, что если мы хотим иметь оценку линейной формы от логарифмов лучше, чем тривиальную, то в теореме $2.2 B$ должно быть достаточно большим. Это также означает, что оценка (2.16) принципиально не изменится, если заменить $\ln (2 e B)$ на $\ln (B F)$ с $F$ порядка $\ll D \omega / A_{n}$.

\section{§ 14. Доказательство теоремы 2.3}

Пусть $p_{k}-k$-е простое число.

Лемма 14.1. Пусть $\alpha_{1}, \ldots, \alpha_{n}$ - мультипликативно независимые рациональные числа. Тогда их можно так перенумеровать, что

$$
h\left(\alpha_{k}\right) \geqslant \ln p_{k}, \quad 1 \leqslant k \leqslant n .
$$

ДокАЗАТЕЛЬСтво. Так как $\alpha_{j}$ мультипликативно независимы, то их разложение на простые множители содержит не меньше $n$ простых чисел. Максимальное из них $p \geqslant p_{n}$. Припишем индекс $n$ числу $\alpha$, содержашему это $p$. Получим $h\left(\alpha_{n}\right) \geqslant \ln p_{n}$. Повторив эту операцию с оставшимися $\alpha$, докажем лемму 14.1.

Примем теперь условия теоремы 2.3. Отметим, что здесь $D=\varkappa=\rho=1$; это упрощает задачу. Поскольку $C_{3}=n$, то мы имеем

$$
C_{1} \geqslant(2+n \ln 2) \frac{n+1}{n^{2}} .
$$


Для $\mathbb{K}=\mathbb{Q}$ мы имеем, что $A_{j} \geqslant h\left(\alpha_{j}\right) \geqslant\left|\ln \alpha_{j}\right|$, значит, $E=1$ удовлетворяет (2.2). Если $E=1, \vartheta \leqslant 1$, то $C_{3}=n$ удовлетворяет (2.3). Из леммы 14.1 получаем неравенства

$$
\begin{gathered}
\Omega \geqslant \Omega_{n}^{*}=\prod_{k=1}^{n} \ln p_{k}, \quad \frac{\Omega}{A_{j}} \geqslant \Omega_{n-1}^{*}, \\
D \vartheta E_{1} \leqslant E_{n}^{*}=\frac{1}{(n-1)} \sum_{k=1}^{n-1} \frac{1}{\ln p_{k}} .
\end{gathered}
$$

ЛЕмма 14.2. (i) Для $C_{1}$ из (14.1) мы имеем

$$
\begin{gathered}
\frac{C_{1}^{n} \Omega_{n}^{*} n e}{2} \geqslant 2.6^{n}, \quad \frac{C_{1}^{n-1} \Omega_{n-1}^{*} n e}{2} \geqslant 2.53^{n-1}, \\
C_{1}^{n-1} \Omega_{n-1}^{*}>1, \quad \frac{E_{n}^{*}}{C_{1}}<1 .
\end{gathered}
$$

(ii) Для $\vartheta=1 /\left(2-2 /\left(n e^{n+1}\right)\right)$ мы имеем

$$
\begin{gathered}
\left(\vartheta C_{1}\right)^{n} \Omega_{n}^{*} \frac{n e}{2 \vartheta} \geqslant 1.39^{n}, \quad\left(\vartheta C_{1}\right)^{n-1} \Omega_{n-1}^{*} \frac{n e}{2 \vartheta} \geqslant 1.37^{n-1}, \\
\left(\vartheta C_{1}\right)^{n-1} \Omega_{n-1}^{*}>0.4, \quad \frac{E_{n}^{*}}{\vartheta C_{1}}<1.33 .
\end{gathered}
$$

ДокАЗАТЕЛЬСТво. Утверждения леммы при $n \leqslant 100$ проверяются непосредственным вычислением. При $n=100$ также проверяем, что

$$
(\ln 2)^{n} \Omega_{n}^{*} \geqslant 2.8^{n}, \quad E_{n}^{*} \leqslant 0.5 \ln 2 .
$$

Для $k \geqslant 100$ также имеем, что $\ln p_{k}>6$. Так как $C_{1}>\ln 2, \vartheta>0.5$, то это доказывает утверждения леммы и при $n \geqslant 100$.

Теперь рассмотрим случай (i) теоремы 2.3. Так как $A_{j} \geqslant h\left(\alpha_{j}\right)$, то $\vartheta=1$ удовлетворяет условию (2.1). Лемма 14.2 (случай (i)) обеспечивает выполнение условий (2.9)-(2.11) теоремы 2.1. Так что случай (i) теоремы 2.3 - это прямое следствие теоремы 2.1 и 2.2 .

Лемма 14.2 также обеспечивает выполнение условий (2.9)-(2.11) в случае (ii) теоремы 2.3 , но $\vartheta=1 /\left(2-2 /\left(n e^{n+1}\right)\right)$ может не удовлетворять условию $(2.1)$. Чтобы доказать случай (ii) теоремы 2.3 , надо повторять доказательство теоремы 2.1, но без упрощения выражения для $\vartheta$. Так что надо проверить, что наше $\vartheta$ удовлетворяет условиям $\S 5$. 
Возьмем тело $\mathscr{W}$ таким же, как в теореме 2.1 , с учетом того, что $\vartheta_{0}^{*}$ определено с помощью (11.7) для $C_{3}=n$. Теперь оценим $h(\bar{\alpha}, \mathscr{W})$. Так как $\alpha_{j} \in \mathbb{Z}$, то вклад неархимедовских нормирований в оценку $h(\bar{\alpha}, \mathscr{W})$ равен

$$
\sum_{\sigma>1} \sup \left\{\sum_{j=1}^{n} \ln \left|\alpha_{j}\right|_{\sigma} w_{j}: \bar{w} \in \mathscr{W}\right\} \leqslant \sum_{\sigma>1} \sum_{j=1}^{n}-\frac{\ln \left|\alpha_{j}\right|_{\sigma}}{2 A_{j}}=\frac{n}{2}
$$

Вклад единственного архимедовского нормирования равен $\vartheta_{0}^{*}$ ввиду (11.2). Значит, в (5.8) мы имеем

$$
\vartheta \leqslant h(\bar{\alpha}, \mathscr{W}) \leqslant \frac{n}{2}+\vartheta_{0}^{*}=n\left(\frac{1}{2}+\frac{\vartheta}{n e^{n+1}}\right)
$$

и простая подстановка показывает, что наше $\vartheta$ согласуется с (11.3). Из леммы 14.2 ,

(ii) также получается (11.11). Это завершает доказательство теоремы 2.3.

\section{Список литературы}

1. Baker A. The theory of linear forms in logarithms // Transcendence theory: advances and applications / Eds. A. Baker, D. W. Masser. London: Academic press, 1977. P. 1-27.

2. Loxton J.H., van der Poorten A.J. Computing the effectively computable bound in Baker's inequality for linear forms in logarithms // Bull. Austral. Math. Soc. 1976. V. 15. P. 33-57.

3. Baker A., Stark H. M. On a fundamental inequality in number theory // Ann. of Math. 1971. V. 94. P. 190-199.

4. Waldschmidt M. A lower bound for linear forms in logarithms // Acta Arithm. 1980. V. 37. P. 257-283.

5. Гельфонд А. О. Трансцендентные и алгебраические числа. М.: Гостехиздат, 1952.

6. Shorey T. N. On linear forms in the logarithms of algebraic numbers // Acta Arithm. 1976. V. 30. P. 27-42.

7. Фельдман Н. И. Об оценке модуля линейной формы от логарифмов некоторых алгебраических чисел // Матем. заметки. 1967. Т. 2. №3. С. 245-256.

8. Матвеев E.M. Линейные формы от значений $G$-функций и диофантовы уравнения // Матем. сб. 1982. Т. 117. № 3. С. 379-396.

9. Stewart C. L., Yu Kunrui. On the abc conjecture // Math. Ann. 1991. V. 291. P. 225-230.

10. De Weger B.M.M. The weighted sum of two $S$-units being a square // Indag. Math. 1990. V. 1. № 2. P. 243-262.

11. Матвеев Е. М. Оценка линейной формы от логарифмов алгебраических чисел: Тез. докл. всесоюзн. конф. "Теория трансцендентных чисел и ее приложения" (Москва, 2-4 февр. 1983). М.: Изд-во МГУ, 1983.

12. Loxton J. H., Mignotte M., van der Poorten A. J., Waldschmidt M. A lower bound for linear forms in the logarithms of algebraic numbers // C. R. Math. Rep. Acad. Sci. Canada. 1987. V. 11. P. 119-124.

13. Blass J., Glass A.M.W., Manski D.K., Meronk D. B., Steiner R.P. Constants for lower bounds for linear forms in the logarithms of algebraic numbers. I, II // Acta Arithm. 1990. V. 55. P. 1-14, 15-22. Corrigenda in: Acta Arithm. 1993. V. 65. P. 383. 
14. Wüstholz G. A new approach to Baker's theorem on linear forms in logarithms. III // New advances in transcendence theory / Ed. A. Baker. Cambridge: Cambridge Univ. Press, 1988. P. 399-410.

15. Philippon P., Waldschmidt M. Lower bounds for linear forms in logarithms // New advances in transcendence theory / Ed. A. Baker. Cambridge: Cambridge Univ. Press, 1988. P. 280-312.

16. Mignotte M., Waldschmidt M. Linear forms in two logarithms and Schneider's method. II // Acta Arithm. 1989. V. 53. P. 251-287.

17. Waldschmidt $M$. Minorations de combinaisons linéaires de logarithmes de nombres algébriques // Canad. J. Math. 1993. V. 45. № 1. P. 176-224.

18. Baker A., Wüstholz G. Logarithmic forms and group varieties // J. reine angew. Math. 1993. V. 442. P. 19-62.

19. Bugeaud Y., Györy K. Bounds for the solutions of Thue-Mahler equations and norm form equations // Acta Arithm. 1996. V. 74. P. 273-292.

20. Матвеев Е. М. О линейных и мультипликативных соотношениях // Матем. сб. 1993. T. 184. № 4. C. $23-40$.

21. Ленг С. Основы диофантовой геометрии. М.: Мир, 1986.

22. Фельдман Н.И. Улучшение оценки линейной формы от логарифмов алгебраических чисел // Матем. сб. 1968. Т. 77. №3. С. 423-436.

23. Van der Poorten A. J. On Baker's inequality for linear forms in logarithms // Math. Proc. Cambridge Philos. Soc. 1976. V. 80. P. 233-248.

24. Лейхтвейс К. Выпуклые множества. М.: Наука, 1985.

25. Касселс Дж. В. С. Введение в теорию диофантовых приближений. М.: ИЛ, 1961.

26. Bombieri E., Vaaler J. On Siegel's Lemma // Invent. Math. 1983. V. 73. P. 11-32. Addendum in: Invent. Math. 1984. V. 75. P. 377.

27. Матвеев E.M. Об арифметических свойствах значений обобщенных биномиальных многочленов // Матем. заметки. 1993. Т. 54. № 4. С. 6-81.

28. Фельдман Н. И. Эффективное степенное усиление теоремы Лиувилля // Изв. АН СССР. Сер. матем. 1972. Т. 35. № 5. С. 973-990.

Московская государственная текстильная

Поступило в редакцию академия им. А.Н. Косыгина.

24.VII.1996

Кафедра высшей математики 